\title{
Where, oh where has the $r$-process gone?
}

\author{
Y.-Z. Qian ${ }^{\mathrm{a}, *}$, G.J. Wasserburg ${ }^{\mathrm{b}}$ \\ ${ }^{a}$ School of Physics and Astronomy, University of Minnesota, Minneapolis, MN \\ 55455, USA \\ ${ }^{\mathrm{b}}$ The Lunatic Asylum, Division of Geological and Planetary Sciences, California \\ Institute of Technology, Pasadena, CA 91125
}

\begin{abstract}
We present a review of the possible sources for $r$-process nuclei ( $r$-nuclei). It is known that there is as yet no self-consistent mechanism to provide abundant neutrons for a robust $r$-process in the neutrino-driven winds from nascent neutron stars. We consider that the heavy $r$-nuclei with mass numbers $A>130$ ( $\mathrm{Ba}$ and above) cannot be produced in the neutrino-driven winds. Nonetheless, the $r$-process and the neutrino-driven winds may be directly or indirectly related by some unknown additional mechanism, which, for example, could provide ejecta with very short dynamic timescales of $\lesssim 0.004 \mathrm{~s}$. This undetermined mechanism must supply a neutron source within the same general stellar sites that undergo core collapse to produce the neutron star. Observational data on low-metallicity stars in the Galactic halo show that sites producing the heavy $r$-nuclei do not produce Fe or any other elements between $\mathrm{N}$ and Ge. Insofar as a forming neutron star is key to producing the heavy $r$-nuclei, then the only possible sources are supernovae resulting from collapse of $\mathrm{O}-\mathrm{Ne}-\mathrm{Mg}$ cores or accretion-induced collapse of white dwarfs, neither of which produce the elements of the Fe group or those of intermediate mass (above $\mathrm{C}$ and N). Observational evidence on $s$ and $r$-nuclei in low-metallicity stars with high $\mathrm{C}$ and $\mathrm{N}$ abundances shows that the $r$-process is also active in binary systems.
\end{abstract}

The nuclei with $A \sim 90-110$ produced by charged-particle reactions (CPR) in the neutrino-driven winds are in general present in metal-poor stars with high or low abundances of heavy $r$-nuclei. The CPR nuclei and the heavy $r$-nuclei are not strongly coupled. Some metal-poor stars show extremely high enrichments of heavy $r$-nuclei and have established that the abundance patterns of these nuclei are universally close to the solar abundance pattern of heavy $r$-nuclei.

Using a template star with high enrichments of heavy $r$-nuclei and another with low enrichments we develop a two-component model based on the abundances of $\mathrm{Eu}$ (from sources for heavy $r$-nuclei) and Fe (from Fe core-collapse supernovae). This model gives very good quantitative predictions for the abundances of all the other elements in those metal-poor stars with $[\mathrm{Fe} / \mathrm{H}] \lesssim-1.5$ for which the Eu and $\mathrm{Fe}$ abundances are known. We attribute the CPR elements such as Sr, Y, and $\mathrm{Zr}$ to reactions in the neutrino-driven winds from a nascent neutron star and the heavy $r$-nuclei to the hypothecated true " $r$-process." The CPR nuclei should be 
produced whenever a neutron star is formed regardless of whether heavy $r$-nuclei are produced or not. Using the two-component model we estimate the yield of the CPR element Sr to be $\sim 10^{-6} M_{\odot}$ for a single neutron star formation event. Selfconsistent astrophysical models are needed to establish that the CPR nuclei are common to the neutron stars produced in both sources for the heavy $r$-nuclei and those for Fe. We show that the observational data appear fully consistent with the two-component model. The specific mechanism and site for the production of heavy $r$-nuclei remains to be found.

Key words: $r$-process nucleosynthesis, abundances in metal-poor stars, Galactic chemical evolution, neutron star formation

PACS: 26.30.+k, 26.45.+h, 97.60.Jd

\section{Introduction}

This article is dedicated to Hans Bethe. The work reported here has its connection to Hans through a strange confluence of events. It has its origins in meteoritics, neutrino physics, and supernovae. Hans and Gerry Brown had been pursuing theories of hypernovae and their inferences on this problem led them to extensive talks with us and greatly stimulated our efforts. Their positive support of our exploration was a stimulating force in all of our work, even when possible direct connections disappeared.

The clear demonstration that the early solar system (ESS) contained many radioactive nuclei with a short to intermediate lifetime required injection from various nucleosynthetic sources. Amongst these nuclei was ${ }^{129} \mathrm{I}\left(\bar{\tau}_{129}=23 \mathrm{Myr}\right)$ discovered by Reynolds (1960). This nuclide cannot be produced by any slow ( " $s$ ") neutron-capture process and requires very high neutron densities for its production. It was clearly to be associated with the " $r$ "-process as enunciated by Burbidge et al. (1957) and Cameron (1957). Measurements of many samples of ESS debris preserved in meteorites showed that the abundance of ${ }^{129} \mathrm{I}$ relative to that of stable ${ }^{127} \mathrm{I}$ was $\left({ }^{129} \mathrm{I} /{ }^{127} \mathrm{I}\right)_{\mathrm{ESS}} \approx 1.0 \times 10^{-4}$ at the time of formation of the solar system. Upon recognizing that this was most plausibly the result of long-term Galactic nucleosynthesis, it followed that the time between the last addition of such $r$-process debris and the formation of the solar system had to be $\sim 10^{8}$ yr (Wasserburg et al., 1960; Schramm \& Wasserburg, 1970 ).

\footnotetext{
* Corresponding author.

Email addresses: qian@physics.umn.edu (Y.-Z. Qian), gjw@gps.caltech.edu (G.J. Wasserburg).
} 
This matter rested almost two decades until clear evidence was found for other short-lived nuclei in the ESS [see the recent review by Wasserburg et al. (2006)]. The various possible stellar nucleosynthetic sources required to produce this inventory of short-lived nuclei led to theoretical models of a wide variety. One possibility, for some of these nuclei, was a low-mass star during its asymptotic giant branch (AGB) stage leading to the formation of a planetary nebula. This potential source had been extensively explored by Gallino et al. (1988) and Käppeler et al. (1990) in studying the $s$-process that is known to take place in such sites. In a collaboration between the Turino Group and the junior author, where he was educated by his Italian colleagues, an AGB model was explored and predictions made of the isotopic yields (Wasserburg et al. 1994$)$. These predictions included yields of ${ }^{182} \mathrm{Hf}\left[\bar{\tau}_{182}=12.8 \pm 0.1 \mathrm{Myr}\right.$ (Vockenhuber et al., 2004)]. It was shown that an AGB source could only produce very little ${ }^{182} \mathrm{Hf}$. This nuclide was subsequently discovered to be also present in the ESS (Lee \& Halliday, 1995; Harper \& Jacobsen, 1996). An AGB source for this nuclide was excluded by the level of its abundance relative to stable ${ }^{180} \mathrm{Hf}$ of $\left({ }^{182} \mathrm{Hf} /{ }^{180} \mathrm{Hf}\right)_{\mathrm{ESS}} \sim 3 \times 10^{-4}$. More recent measurements have now established a reliable value of $\left({ }^{182} \mathrm{Hf} /{ }^{180} \mathrm{Hf}\right)_{\mathrm{ESS}}=(1.00 \pm 0.08) \times 10^{-4}(\mathrm{Yin}$ et al., 2002; Kleine et al., 2002). However, the difficulty for an AGB source to account for the ${ }^{182} \mathrm{Hf}$ in the ESS is only slightly alleviated. The major problem persisted - this nuclide must be produced in an $r$-process and cannot be made effectively in an $s$-process.

This led to the following dilemma: both ${ }^{129} \mathrm{I}\left(\bar{\tau}_{129}=23 \mathrm{Myr}\right)$ and ${ }^{182} \mathrm{Hf}\left(\bar{\tau}_{182}=\right.$ $12.8 \mathrm{Myr})$ could not have been produced by the same $r$-process source $(r$ source) in order to account for their measured relative abundances in the ESS. This results from the simple fact that there is a factor of 1.8 difference in their lifetimes. For example, consider a model of continuous nucleosynthesis over a Galactic timescale $T_{\mathrm{UP}} \sim 10^{10}$ yr with uniform (number) production rates $P_{i}$ for nuclide $i$. In this case the interstellar medium (ISM) would have an inventory of $P_{\mathrm{S}} T_{\mathrm{UP}}$ for a stable nucleus $(\mathrm{S})$ and $P_{\mathrm{R}} \bar{\tau}_{\mathrm{R}}$ for a radioactive one $(\mathrm{R})$. If this uniform production is followed by a period of no production for a time $\Delta$ prior to the formation of the solar system, then the abundance of $\mathrm{R}$ in the ESS is diminished by a factor of $\exp \left(-\Delta / \bar{\tau}_{\mathrm{R}}\right)$. For ${ }^{129} \mathrm{I}$ we have

$$
\left(\frac{{ }^{129} \mathrm{I}}{{ }^{127} \mathrm{I}}\right)_{\mathrm{ESS}}=\left(\frac{P_{129}}{P_{127}}\right)\left(\frac{\bar{\tau}_{129}}{T_{\mathrm{UP}}}\right) \exp \left(-\frac{\Delta}{\bar{\tau}_{129}}\right) .
$$

If the ratio of the production rates is of order unity, then the above equation gives $\Delta \sim 72$ Myr. For this $\Delta$ and assuming $P_{182} / P_{180} \sim 1$, a similar equation for ${ }^{182} \mathrm{Hf}$ would give $\left({ }^{182} \mathrm{Hf} /{ }^{180} \mathrm{Hf}\right)_{\mathrm{ESS}} \sim 4.6 \times 10^{-6}$, much smaller than the measured value of $1.0 \times 10^{-4}$. As there is no basis for considering a large production ratio $P_{182} / P_{180} \sim 22$ to make up for this gross difference, it follows that ${ }^{129} \mathrm{I}$ and ${ }^{182} \mathrm{Hf}$, both $r$-process nuclei ( $r$-nuclei), cannot be produced in 
the same type of event. As pointed out by Wasserburg et al. (1996), this fact requires at least two types of $r$-process event and must be related to the differences between the site of production for the light $r$-nuclei with mass numbers $A \lesssim 130$ and that for the heavy ones with $A>130$. These authors also noted that elemental abundances in low-metallicity stars formed in the early Galaxy would be highly susceptible to the particular type of $r$-source contaminating their birth medium. The study of metal-poor stars in the Galactic halo, as was already being pursued by Chris Sneden and his colleagues, would clearly be of aid in testing this inference. Such studies indeed prove to be of great importance in establishing the diversity of $r$-sources.

For almost forty years there had been a general preference for considering the $r$-process to be associated with a single type of astrophysical source that has an appropriate dynamic timescale 11 The site usually discussed was an explosive environment such as some region inside a core-collapse supernova. The evolution and nucleosynthesis of massive stars associated with such supernovae is discussed in the book by Arnett (1996) and is reviewed in a recent paper by Woosley et al. (2002). Following the seminal paper by Bethe \& Wilson (1985) on the neutrino-driven supernova mechanism, the material ejected by neutrino heating from the vicinity of a nascent neutron star - the neutrino-driven wind - was considered the most promising site for the $r$-process (Woosley \& Baron, 1992; Woosley \& Hoffman, 1992; Meyer et al., 1992; Howard et al., 1993; Takahashi et al., 1994; Woosley et al., 1994). For many workers in the field, the possibility of multiple $r$-sources was not readily accepted. Although it was generally understood that a superposition of yield patterns each covering a different mass range is required to reproduce the overall solar $r$-process abundance pattern ( $r$-pattern) in the absence of fission cycling [e.g., Kratz et al. (1993)], it was preferred to consider this superposition as the result of mixing within a single astrophysical environment. For example, it was regarded as a major success when the model of Woosley et al. (1994) appeared to produce

$\overline{1}$ Neutron star mergers have often been cited as a site for the $r$-process. Some workers have studied the possibility that these events are the source for the heavy $r$-nuclei with $A>130$ [e.g., Freiburghaus et al. (1999b)]. However, the problem is that these events are too infrequent (by a factor of $\gtrsim 1000$ ) compared to corecollapse supernovae. If neutron star mergers were the source for heavy $r$-nuclei, then enrichment in these nuclei would not occur until the ISM had already been substantially enriched in Fe by Fe core-collapse supernovae from progenitors of $\sim$ $12-25 M_{\odot}($ Qian, 2000; Argast et al., 2004). This is in contradiction to the existence of stars with very low Fe abundances but highly enriched in heavy $r$-nuclei (see Section 4.1). In addition, the observations so far show that high enrichments of heavy $r$-nuclei are always accompanied by enrichments of the nuclei with $A \sim 90$ 110 at a roughly comparable level (see Sections 4.3 and 6.2). However, it has not been demonstrated that the latter nuclei can be produced in neutron star mergers. For the above reasons, we will not discuss neutron star merger models for the $r$ process further. 
an integrated yield pattern from a single supernova that closely resembles the solar $r$-pattern with peaks at $A \sim 130$ and 195. The compelling evidence obtained later from spectroscopic data of very high quality on several key halo stars of ultra-low metallicities has brought the issue of diverse $r$-sources to the forefront of theoretical consideration.

When the ${ }^{129} \mathrm{I}$ and ${ }^{182} \mathrm{Hf}$ dilemma appeared, the matter was a source of lunchtime discussions in the Athenaeum at Caltech with Hans and Gerry. It was also imposed on Rose who has gracefully endured this type of "social" conversation for many years. Hans, of course, on first hearing the dilemma would go on about the nuclear details of ${ }^{129} \mathrm{I}$ and ${ }^{182} \mathrm{Hf}$ without any books — just from his incredible mind. Awesome!

The senior author was a research fellow in Petr Vogel's group at Caltech around the time when the ${ }^{129} \mathrm{I}$ and ${ }^{182} \mathrm{Hf}$ dilemma was first being discussed. He had just finished a detailed study on the neutrino-driven wind with Stan Woosley, which showed that the conditions in the wind fall short of what is required for an $r$-process (Qian \& Woosley, 1996). It did not appear that a straightforward ab initio solution to the $r$-process problem was within reach. On the other hand, a phenomenological approach based on the available observational data might offer some helpful guidance. The ${ }^{129} \mathrm{I}$ and ${ }^{182} \mathrm{Hf}$ dilemma seemed to provide a good starting point for this approach. In an initial effort, Qian et al. (1998) proposed two types of supernova $r$-process event: the $H$ type producing mainly the heavy $r$-nuclei with $A>130$ and the $L$ type producing mainly the light ones with $A \lesssim 130$. The overall solar $r$-pattern was considered to have resulted from a mixture of these two types of event. The meteoritic data on ${ }^{129} \mathrm{I}$ and ${ }^{182} \mathrm{Hf}$ could also be accounted for by requiring that the last event contributing $r$-nuclei to the solar system be of the $H$ type. Based on later observations of abundances in metal-poor stars, the authors of this report developed a phenomenological model with more detailed description of the $H$ and $L$ events (Qian \& Wasserburg, 2001, 2002). This model makes quantitative predictions for elemental abundances that are in good agreement with the observations. In this report, we will present inferences that we drew from the earlier data and then show some changes that must follow from the newer highquality observational results obtained by many groups. Our basic conclusions reached earlier appear to remain valid.

\section{The phenomenological $r$-process}

The logarithm of the solar " $r$ "-process abundance (" $r$ "-abundance) $N_{\odot, r}(A)$ of a nuclide with mass number $A$ is shown as a function of $A$ in Figure 1 . The 

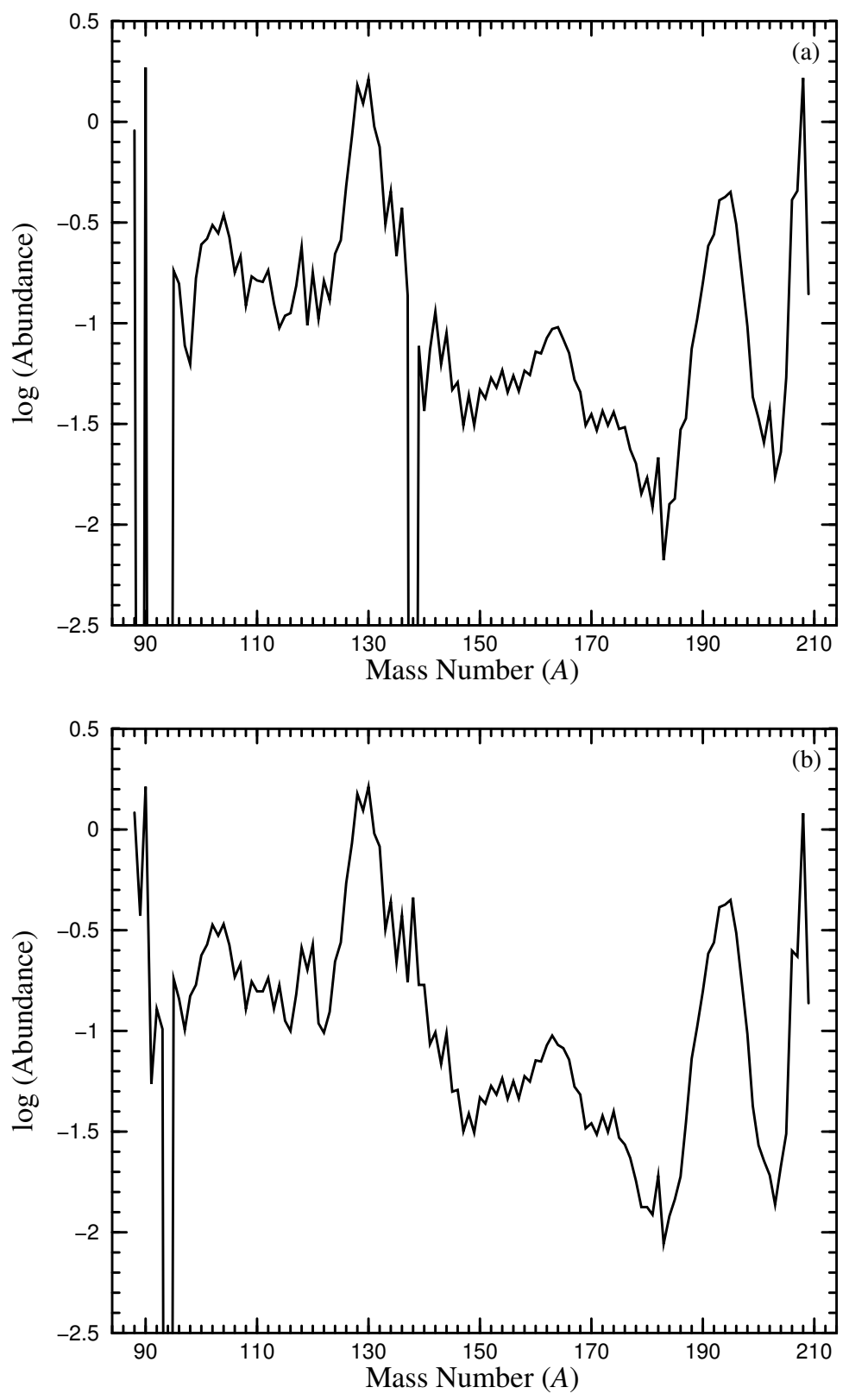

Fig. 1. The logarithm of the solar " $r$ "-abundance $N_{\odot, r}=N_{\odot}-N_{\odot, s}$ (normalized so that the elemental abundance of $\mathrm{Si}$ is $10^{6}$ ) as a function of $A$. The $s$-process contribution $N_{\odot, s}$ is taken from Arlandini et al. (1999) and calculated using (a) a phenomenological model or (b) a blend of 1.5 and $3 M_{\odot}$ AGB sources with an initial metallicity of half the solar value.

value $N_{\odot, r}(A)$ is obtained from

$$
N_{\odot, r}(A)=N_{\odot}(A)-N_{\odot, s}(A)
$$


where $N_{\odot}(A)$ is the total solar abundance of the nuclide derived from meteoritic and spectroscopic measurements [normalized so that the elemental abundance of Si is $10^{6}$, see e.g., Anders \& Grevesse (1989)], and $N_{\odot, s}(A)$ is the $s$-process contribution to this nuclide (the solar main $s$-component) calculated using a phenomenological model (Fig. 1 1 a) or a blend of 1.5 and $3 M_{\odot}$ AGB sources with an initial metallicity of half the solar value (Fig. 1b). The $s$-process contributions used are taken from Arlandini et al. (1999). The total solar abundances are rather well determined, and so are the majority of the solar " $r$ "-abundances. Neither sets of abundances have changed drastically in forty years (except for $\mathrm{Fe}$ ) although both have been regularly subject to revisions. There is a problem with attributing $N_{\odot, r}$ obtained by $s$-process subtraction for nuclei with $A \sim 90-110$ to the $r$-process. This will be discussed in Section 3 ,

Clearly, the solar inventory is the result of contributions from many stellar sources over Galactic history. Due to mixing of nucleosynthetic products from different sources in the ISM over this long history, the patterns for the gross abundances of a wide range of elements in other stars with approximately solar metallicity are observed to be close to the solar pattern. It is well recognized that this apparent "universality" does not imply the existence of a single process for making all the nuclei in nature. However, when only production by rapid neutron capture is considered, it is tempting to regard the solar $r$-pattern as a truly universal yield pattern of every single $r$-process event. Theoretically speaking, it is possible to reproduce the solar $r$-pattern with peaks at both $A \sim 130$ and 195 (see Fig. 11) by fission cycling in a single environment with extremely abundant supply of neutrons [e.g., Seeger et al. (1965)]. However, this would most likely couple the production of ${ }^{129} \mathrm{I}$ and ${ }^{182} \mathrm{Hf}$, in contradiction to the meteoritic data discussed in Section 1. In addition, so far there is no selfconsistent astrophysical model that can demonstrate the occurrence of fission cycling [the model of Freiburghaus et al. (1999b) is of parametric nature]. There have also been studies aiming to reproduce the overall solar $r$-pattern from a single site without fission cycling [e.g., Woosley et al. (1994)]. However, these studies are either parametric or unsuccessful [the nonparametric model of Woosley et al. (1994) has several serious deficiencies, see e.g., Meyer (1995); Qian \& Woosley (1996)].

An $r$-process requires an environment of high neutron (number) density with a large abundance of neutrons relative to the seed nuclei capturing them. The theory of the $r$-process has four components: (1) what are the seed nuclei and how are they provided? (2) how do temperature, density, and neutron abundance evolve with time during the $r$-process? (3) what are the properties of the nuclei participating in the $r$-process and the rates of the relevant reactions? (4) how many types of astrophysical sources with distinct $r$-process yield $(r$ yield) patterns are there to account for the chemical evolution of $r$-nuclei in the Galaxy and elsewhere? The third component is in the domain of nuclear 
physics and is crucial to the determination of the exact $r$-yield pattern produced by a specific astrophysical source. We will not discuss this component here. Instead, we address some aspects of the first and second components and focus on the fourth considering observations of metal-poor stars and data on the solar system.

Nearly all $r$-process models are of parametric nature and fall into two categories: one is self-contained in that the first and second components listed above are addressed within the model [e.g., Hoffman et al. (1997); Meyer \& Brown (1997); Freiburghaus et al. (1999a)], while the other assumes a seed nucleus and subjects it to neutron bombardment at constant neutron density and temperature for some time [e.g., Kratz et al. (1993)]. In the absence of fission cycling, both categories of models require a superposition of $r$-yield patterns produced under different conditions to reproduce the overall solar $r$ pattern, but neither can determine whether this superposition is achieved by combining the $r$-nuclei produced in different regions at different times within a single astrophysical source or by mixing the $r$-nuclei produced by different types of source in the ISM.

The requirement of superposition to reproduce the solar $r$-pattern without fission cycling can be understood from the following simplified description of the $r$-process. Consider an $r$-process starting with a ratio $n / s$ of the total number of free neutrons to that of the seed nuclei. As all the neutrons will be captured, the equation governing this $r$-process is

$$
\left\langle A_{s}\right\rangle+n / s=\left\langle A_{r}\right\rangle
$$

where $\left\langle A_{s}\right\rangle$ is the average mass number of the seed nuclei and $\left\langle A_{r}\right\rangle$ that of the final $r$-nuclei produced. It follows that for $\left\langle A_{s}\right\rangle \sim 90, n / s \sim 40$ is required to produce an abundance peak at $A \sim 130$. From the same seed distribution, to produce an abundance peak at $A \sim 195$ would require $n / s \sim 100$. It is immediately evident that the solar $r$-pattern with peaks at both $A \sim 130$ and 195 (see Fig. 1) can only be reproduced by a superposition of $r$-yield patterns corresponding to a range of $n / s$. For illustration, we assume that a single kind of seed nucleus with mass number $A_{s}=90$ is present at time $t=0$ and that the $r$-process subsequently passes through a fixed set of nuclei. We show the progress of the $r$-process for different $n / s$ values in Figure 2. For $n / s=44$ all the neutrons have been captured by $t=0.78 \mathrm{~s}$, resulting in an $r$-yield pattern dominated by the peak at $A \sim 130$ (panel labeled " $t=0.78 \mathrm{~s}$ "). By comparison, for a higher $n / s=86$ many neutrons remain to be captured at $t=0.78 \mathrm{~s}$ and rapid neutron capture continues until $t=1.68 \mathrm{~s}$, leading to major production of the nuclei in the peak at $A \sim 195$ and beyond (panel labeled " $t=1.68 \mathrm{~s}$ "). The solar $r$-pattern with peaks at both $A \sim 130$ and 195 can then be reproduced, for example, by an appropriate superposition of the $r$-yield patterns for $n / s=44$ and 86 (Qian et al. 1998). 

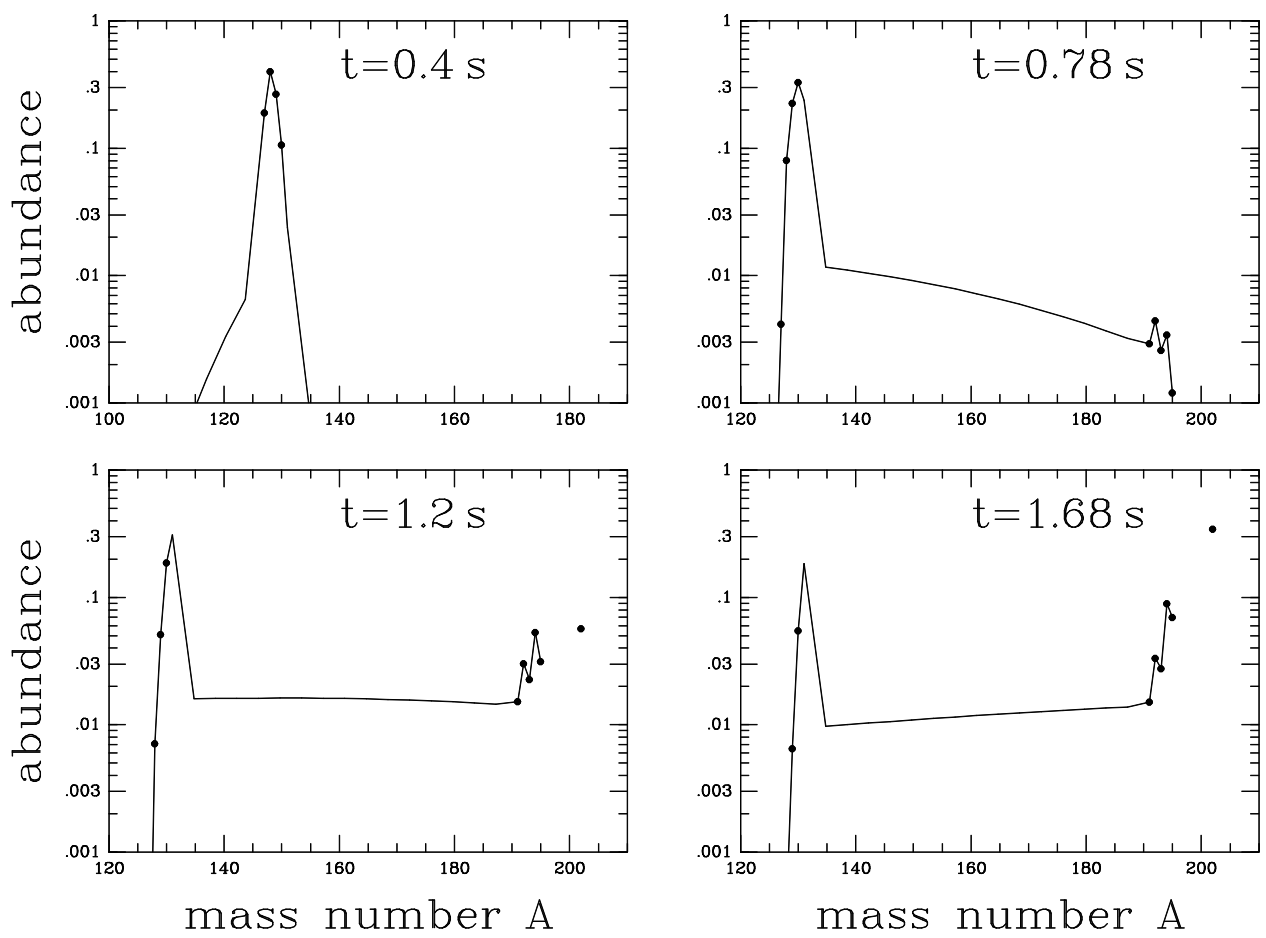

Fig. 2. Illustration of the progress of the $r$-process. It is assumed that a single kind of seed nucleus with $A_{s}=90$ is present at $t=0$ and that the $r$-process passes through a fixed set of nuclei. Rapid neutron capture stops at $t=0.78 \mathrm{~s}$ for $n / s=44$ and at $t=1.68 \mathrm{~s}$ for $n / s=86$. The panels labeled by these times give the yield patterns for these two $n / s$ values. Other panels represent intermediate stages before all neutrons are captured.

To summarize, the solar $r$-pattern represents a superposition of $r$-yield patterns produced with a range of $n / s$ (some variation in the seed nuclei is also possible) in the absence of fission cycling. Meteoritic data on ${ }^{129} \mathrm{I}$ and ${ }^{182} \mathrm{Hf}$ suggest that this superposition was accomplished by mixing the $r$-nuclei produced by two distinct types of source in the ISM: one has high $n / s$ and produces mainly the heavy $r$-nuclei with $A>130$ while the other has lower $n / s$ and produces mainly the light $r$-nuclei with $A \lesssim 130$. The issue of two distinct $r$ sources will be discussed further in Section 4 in connection with observations of metal-poor stars. As mentioned above, it is possible to produce simultaneously the peaks at both $A \sim 130$ and 195 in the solar $r$-pattern with fission cycling. In this case, the average mass number $\left\langle A_{r}\right\rangle$ of the nuclei produced is determined by the $r$-process flow between the fission fragments and the nuclei undergoing fission. The total abundance of the nuclei produced is essentially governed by the available neutron supply. Clearly, Equation (3) does not apply to the case of fission cycling as the mass numbers of the seed nuclei are no longer pertinent. The possible role of fission cycling in $r$-process 
nucleosynthesis will be discussed in Section 4.6. We now turn to a class of astrophysical models for heavy element synthesis that may be closely related to the $r$-process.

\section{Heavy element synthesis in neutrino-driven winds}

The formation of a neutron star in a core-collapse supernova results in the emission of intense flux of $\nu_{e}, \bar{\nu}_{e}, \nu_{\mu}, \bar{\nu}_{\mu}, \nu_{\tau}$, and $\bar{\nu}_{\tau}$ as the neutron star releases its enormous gravitational binding energy of several $10^{53}$ erg during the first $\sim 20 \mathrm{~s}$ of its life. As $\nu_{e}$ and $\bar{\nu}_{e}$ pass through the "atmosphere" of free neutrons and protons surrounding the neutron star, some of them are captured via the reactions

$$
\begin{gathered}
\nu_{e}+n \rightarrow p+e^{-}, \\
\bar{\nu}_{e}+p \rightarrow n+e^{+} .
\end{gathered}
$$

The heating of the atmosphere mainly by the above reactions makes it expand, thereby forming a neutrino-driven wind from the neutron star. The heating occurs mostly within a few neutron star radii and becomes inefficient at larger distance due to the decrease in the neutrino flux and the increase in the wind velocity. Therefore, the wind material expands approximately adiabatically above several neutron star radii. The profuse production of neutrons via the reaction in Eq. (5) in the vicinity of the neutron star, although countered by destruction via the reaction in Eq. (4), suggests the possibility that the neutrino-driven wind may be associated with the $r$-process.

The adiabatic expansion of a mass element in the wind is governed by three key parameters: the entropy $S$, the dynamic timescale $\tau_{\text {dyn }}$, and the electron fraction $Y_{e}$. The electron fraction specifies the initial number fractions of free neutrons and protons as $Y_{n}=1-Y_{e}$ and $Y_{p}=Y_{e}$, respectively, when the mass element is near the neutron star. The dynamic timescale $\tau_{\text {dyn }}$ controls how fast the temperature decreases as the element expands, and $S$ determines the density at a given temperature. These parameters are determined in turn by the state of the neutron star that is defined by its mass $M_{\mathrm{NS}}$ and radius $R_{\mathrm{NS}}$ as well as its neutrino emission characteristics when the mass element is ejected [e.g., Qian \& Woosley (1996); Thompson et al. (2001)]. The amount of material ejected with a specific set of $S, \tau_{\text {dyn }}$, and $Y_{e}$ depends on how long the neutron star stays in the corresponding state. During the period of interest, $M_{\mathrm{NS}}$ remains essentially constant but $R_{\mathrm{NS}}$ decreases substantially, the neutrino luminosity decreases drastically, and the neutrino energy spectra can change substantially. In any case, given the evolution of $R_{\mathrm{NS}}$ and the neutrino emission characteristics of a neutron star, one can calculate the amounts of material 
ejected with various sets of $S, \tau_{\text {dyn }}$, and $Y_{e}$ during this evolution. For a mass element with a specific set of these parameters, nuclear reactions during its expansion produce a particular set of heavy nuclei with specific abundances. The total production of heavy nuclei in the neutrino-driven wind is then an integral over the evolution of the neutron star.

We now consider some examples of nucleosynthesis associated with the expansion of a mass element in the wind from a neutron star with $M_{\mathrm{NS}}=1.4 M_{\odot}$. The evolution of the radius and neutrino emission characteristics of this neutron star is taken from Woosley et al. (1994). Consider the epoch for which $R_{\mathrm{NS}}=10 \mathrm{~km}$ and the neutrino energy spectra change slowly. It is convenient to use the neutrino luminosity $L_{\nu}(t)$ as a function of time $t$ to represent the evolution of the neutron star during this epoch. At a given time, a mass element is ejected and processed as it moves outward until all nuclear reactions cease. At a later time, another element is ejected and moves outward being processed at a different neutrino luminosity until all nuclear reactions cease. There are two timescales involved: the dynamic timescale $\tau_{\text {dyn }}$ for a mass element to move out and the evolutionary timescale $\tau_{\nu}$ over which $L_{\nu}$ changes significantly. Typically we have $\tau_{\text {dyn }} \ll \tau_{\nu}$. Thus, the conditions and the resulting nucleosynthesis would be similar for mass elements ejected within an interval $\Delta t \lesssim \tau_{\nu}$

We pick two times $t_{A}$ and $t_{B}$ during the evolution of the above neutron star. For $L_{\nu}\left(t_{A}\right)=3 \times 10^{51} \mathrm{erg} / \mathrm{s}$ per species, the mass element is ejected with $S \sim 74$ (in units of Boltzmann constant per baryon), $\tau_{\mathrm{dyn}}=0.024 \mathrm{~s}$, and $Y_{e}=0.465$; for $L_{\nu}\left(t_{B}\right)=10^{51} \mathrm{erg} / \mathrm{s}$ per species, the conditions change to $S \sim 87, \tau_{\text {dyn }}=0.066 \mathrm{~s}$, and $Y_{e}=0.372$ (Qian \& Woosley, 1996). The difference in $\tau_{\text {dyn }}$ is mainly due to the factor of 3 difference in $L_{\nu}$ while that in $Y_{e}$ mostly results from the change in the neutrino energy spectra. For the mass element ejected at $t_{A}$, nucleosynthesis predominantly produces ${ }^{88} \mathrm{Sr},{ }^{89} \mathrm{Y}$, and ${ }^{90} \mathrm{Zr}$ [see Fig. 1 in Hoffman et al. (1997)]. For the mass element ejected at $t_{B}$, the dominant products are ${ }^{96} \mathrm{Zr},{ }^{98,100} \mathrm{Mo},{ }^{101,104} \mathrm{Ru},{ }^{105,110} \mathrm{Pd}$, and ${ }^{107} \mathrm{Ag}$ [see Fig. 2 in Hoffman et al. (1997). Note that ${ }^{107} \mathrm{Ag}$ was made as ${ }^{107} \mathrm{Mo}$, which decays through ${ }^{107} \mathrm{Pd}$ with $\bar{\tau}_{107}=9.4 \mathrm{Myr}$, see Woosley \& Hoffman (1992)]. However, for both cases the heavy nuclei are produced by the so-called $\alpha$ process involving charged-particle reactions (Woosley \& Hoffman, 1992), not by rapid neutron capture. In fact, by the time charged-particle reactions cease due to the prohibitive Coulomb barrier at a temperature $T \sim 2-3 \times 10^{9} \mathrm{~K}$, few neutrons are left for each heavy nucleus so that no significant processing by rapid neutron capture can occur subsequently (Hoffman et al., 1997).

The above results are representative of nucleosynthesis in the neutrino-driven wind from a $1.4 M_{\odot}$ neutron star. While nuclei with $A \sim 90-110$ can be produced by the $\alpha$-process, there are too few neutrons left at the end of the $\alpha$-process to drive a subsequent $r$-process. As the higher neutrino luminosity 
$L_{\nu}\left(t_{A}\right)$ is more efficient in ejecting the material than the lower $L_{\nu}\left(t_{B}\right)$, the total production integrated over the evolution of the neutron star is dominated by ${ }^{88} \mathrm{Sr},{ }^{89} \mathrm{Y}$, and ${ }^{90} \mathrm{Zr}$ (Woosley et al., 1994). If we consider a more massive neutron star with $M_{\mathrm{NS}}=2 M_{\odot}$, the production at earlier times during its evolution is similar to the $1.4 M_{\odot}$ case. However, the wind has significantly higher $S$ at later times. For example, the neutron star state with $R_{\mathrm{NS}}=$ $10 \mathrm{~km}$ and $L_{\nu}=6 \times 10^{50} \mathrm{erg} / \mathrm{s}$ per species corresponds to $S \sim 140, \tau_{\mathrm{dyn}}=$ $0.11 \mathrm{~s}$, and $Y_{e}=0.354$ (Qian \& Woosley, 1996). For these parameters, the $\alpha$ process dominantly produces ${ }^{97,100} \mathrm{Mo},{ }^{102,104} \mathrm{Ru},{ }^{103} \mathrm{Rh},{ }^{105,108,110} \mathrm{Pd},{ }^{107,109} \mathrm{Ag}$, and ${ }^{111} \mathrm{Cd}$. In addition, a significant part of the nuclear flow has reached $A=$ 123-125, signaling an incipient $r$-process [see Fig. 6 in Hoffman et al. (1997)]. However, even in this case there are also only $\lesssim 10$ neutrons available for each heavy nucleus produced by the $\alpha$-process.

One caveat for the $2 M_{\odot}$ neutron star case is that there is no self-consistent calculation of either its neutrino emission characteristics or the evolution of its radius. In the above example, we have simply assumed $R_{\mathrm{NS}}=10 \mathrm{~km}$ and taken the neutrino energy spectra corresponding to $L_{\nu}=6 \times 10^{50} \mathrm{erg} / \mathrm{s}$ per species for the $1.4 M_{\odot}$ case. Many studies of the $r$-process [see the recent review by Wanajo \& Ishimaru (2006)] are of this parametric nature and thus lack self-consistency. It may be quite possible that a self-consistent calculation of the evolution of a $2 M_{\odot}$ neutron star may give rise to an $r$-process producing nuclei with $A \sim 130$ during some part of its evolution. For production of $r$ nuclei with $A \sim 195$, there must be $\sim 100$ neutrons left for each heavy nucleus produced when the $\alpha$-process ceases at $T \sim 2-3 \times 10^{9} \mathrm{~K}$. This requires much more extreme conditions in either entropy or dynamic timescale as shown in Figure 3. Given the typical conditions of $S \lesssim 140, \tau_{\mathrm{dyn}} \sim 0.01-0.1 \mathrm{~s}$, and $0.35 \lesssim Y_{e}<0.5$ in the neutrino-driven wind [e.g., Qian \& Woosley (1996); Thompson et al. (2001)], it appears that there is no clear case for sufficient neutrons to produce $r$-nuclei with $A \sim 195$ in the wind. We here consider that the neutrino-driven wind is not directly responsible for the production of the heavy $r$-nuclei with $A>130$.

Insofar as the neutrino-driven wind model is basically correct, then the need for a bridge between the $\alpha$-process in the wind and the real $r$-process remains the key issue. There is some confusion in the literature with regard to the "light $r$ nuclei" with $A \sim 90-110$. Insofar as they mostly represent the products of the $\alpha$-process involving charged-particle reactions that would precede any true $r$-process, they should not be considered or lumped with the true $r$-nuclei produced by rapid neutron capture. We will henceforth refer to the nuclei with $A \sim 90-110$ produced by charged-particle reactions (the $\alpha$-process) in the neutrino-driven wind as "CPR" nuclei. The corresponding elements are $\mathrm{Sr}, \mathrm{Y}, \mathrm{Zr}, \mathrm{Nb}, \mathrm{Mo}, \mathrm{Ru}, \mathrm{Rh}, \mathrm{Pd}, \mathrm{Ag}$, and $\mathrm{Cd}$.

The mechanism of the $\alpha$-process for producing CPR nuclei in the neutrino- 


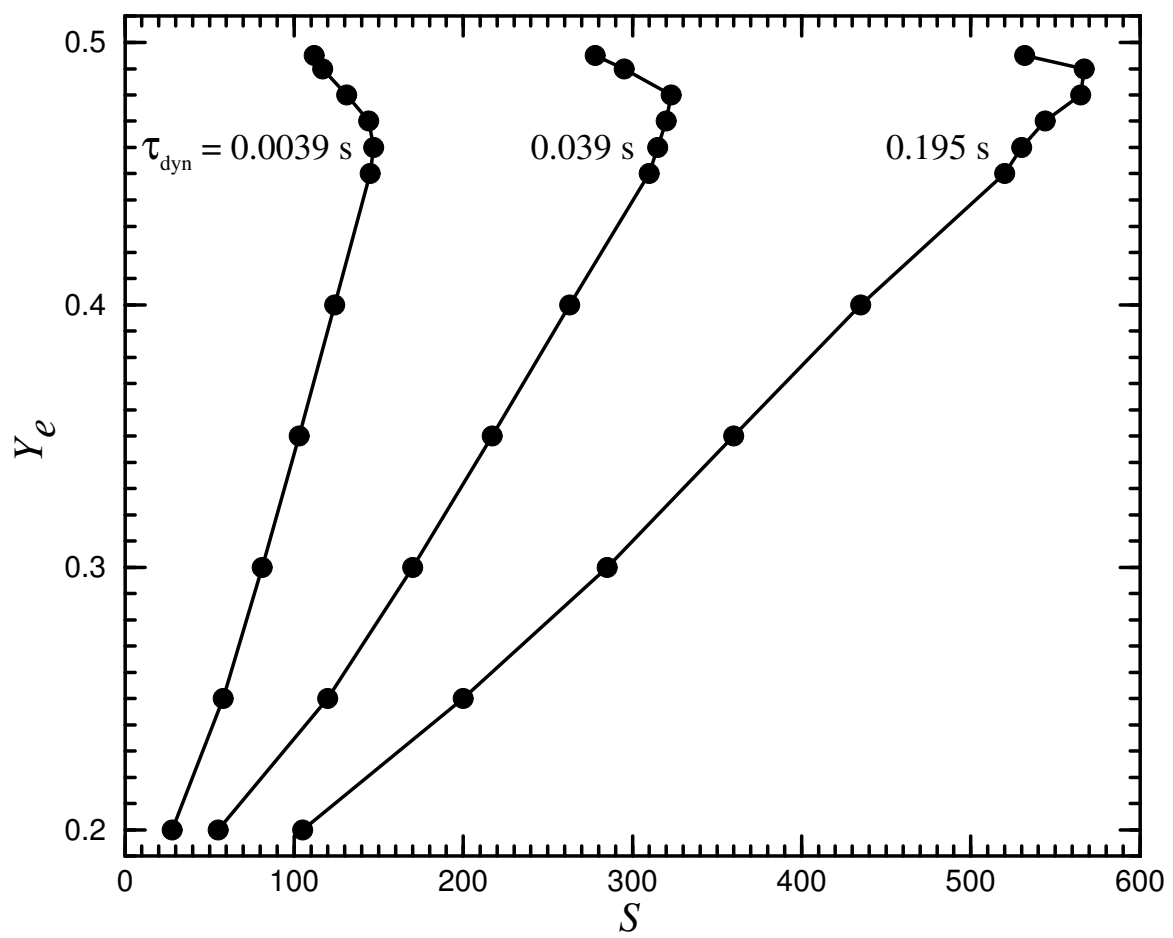

Fig. 3. Combinations of $S, Y_{e}$, and $\tau_{\text {dyn }}$ required to produce $r$-nuclei with $A \sim 195$ in an adiabatically expanding mass element such as that in the neutrino-driven wind. Three choices of $\tau_{\text {dyn }}$ are shown [see Hoffman et al. (1997) for discussion and more results]. Note that for $S \lesssim 140$ and $0.35 \lesssim Y_{e}<0.5$ typical of the neutrino-driven wind, the high $n / s$ required for production of $r$-nuclei with $A \sim 195$ can only be obtained with very short dynamic timescales $\tau_{\text {dyn }} \lesssim 0.0039$ s. However, typical values for the wind are $\tau_{\text {dyn }} \sim 0.01-0.1 \mathrm{~s}$ [e.g., Qian \& Woosley (1996); Thompson et al. (2001)].

driven wind is quite well understood (Woosley \& Hoffman, 1992). However, the quantitative yields of these nuclei depend on the detailed evolution of the radius and neutrino emission characteristics of nascent neutron stars as discussed above. To our knowledge, so far there is only one calculation that follows the evolution of a $1.4 M_{\odot}$ neutron star during the first $\sim 20$ s of its life (Woosley et al., 1994). There are also significant uncertainties in calculating the neutrino luminosity and energy spectra, which have a major impact on $Y_{e}$ in the wind (Qian et al., 1993). Due to these issues, there are no robust predictions for the yields of CPR nuclei from the neutrino-driven wind. However, it seems clear from the available calculations that Sr, Y, and Zr must 
be the dominant products [e.g., Woosley et al. (1994)]. Using the available data on metal-poor stars (Hill et al., 2002; Johnson \& Bolte, 2002; Aoki et al., 2005; Barklem et al., 2005; Otsuki et al., 2006; Ivans et al., 2006), we show $\log \epsilon(\mathrm{Sr}) \equiv \log (\mathrm{Sr} / \mathrm{H})+12$, where $(\mathrm{Sr} / \mathrm{H})$ is the number ratio of Sr to $\mathrm{H}$ observed in a star, as a function of $[\mathrm{Fe} / \mathrm{H}] \equiv \log (\mathrm{Fe} / \mathrm{H})-\log (\mathrm{Fe} / \mathrm{H})_{\odot}$ in Figure 4 a. It is clear that there is a large scatter in $\log \epsilon(\mathrm{Sr})$ at any fixed $[\mathrm{Fe} / \mathrm{H}]$ over the wide range $-3.5 \lesssim[\mathrm{Fe} / \mathrm{H}]<-2.5$. Therefore, there is no correlation between $\mathrm{Sr}$ and $\mathrm{Fe}$ abundances at such low metallicities. In contrast, $\log (\mathrm{Sr} / \mathrm{Y})$ and $\log (\mathrm{Zr} / \mathrm{Y})$ are rather constant over the entire range $-3.5 \lesssim[\mathrm{Fe} / \mathrm{H}] \lesssim-1.5$

for the data shown and cluster closely around the values for CS 22892-052 [Sneden et al. (2003), see Figs. 4b and 4c]. As will be discussed in Section 4 , CS 22892-052 has $[\mathrm{Fe} / \mathrm{H}]=-3.1$ but is greatly enriched in heavy $r$-nuclei. The relationship between Sr, Y, and Zr relative to Fe shown in Figures $4 \mathrm{~b}$ and 4 c can be understood if CPR nuclei are produced with an approximately fixed yield pattern in the neutrino-driven wind whenever a neutron star is formed. However, it follows from Figure 4 a that the CPR elements are not always produced with Fe (see Sections 6.1 and 6.2).

There are three possible venues for making a neutron star: (1) collapse of an Fe core produced by progenitors of $\sim 11-25 M_{\odot},(2)$ collapse of an $\mathrm{O}-\mathrm{Ne}-\mathrm{Mg}$ core produced by progenitors of $\sim 8-10 M_{\odot}$, and (3) accretion-induced collapse (AIC) of a white dwarf in binary systems. These venues will be referred to as Fe core-collapse supernovae (SNe), O-Ne-Mg core-collapse SNe, and AIC events, respectively (we also refer to all three generically as core-collapse SNe for convenience). Only the first venue - Fe core-collapse SNe - can produce Fe (see Sec. 4.4). If a similar amount of CPR nuclei is produced per event for all three venues, there will be no correlation between these nuclei and Fe at low $[\mathrm{Fe} / \mathrm{H}]$ values for which the abundances in stars would be highly susceptible to the particular type of event contaminating their birth medium. In addition, if CPR nuclei are always produced with nearly fixed yield ratios, then the approximate constancy of $\log (\mathrm{Sr} / \mathrm{Y})$ and $\log (\mathrm{Zr} / \mathrm{Y})$ over the wide range of $[\mathrm{Fe} / \mathrm{H}]$ shown in Figures $4 \mathrm{~b}$ and 4 c can also be understood. In summary, the data on metal-poor stars provide some strong indirect evidence for the role of the neutrino-driven wind in producing CPR nuclei. The relationship between these nuclei and the true $r$-nuclei will be discussed in Sections 4.3, 6.1, and 6.2 .

\section{Critical astronomical observations on the $r$-rocess sites}

It is most plausible that stars of very low metallicities (as determined by $[\mathrm{Fe} / \mathrm{H}]$ ) would have formed when the ISM was only slightly enriched in the products of stellar nucleosynthesis. In particular, the $s$-process contributions from low-mass AGB stars to the ISM would not have occurred during the first 

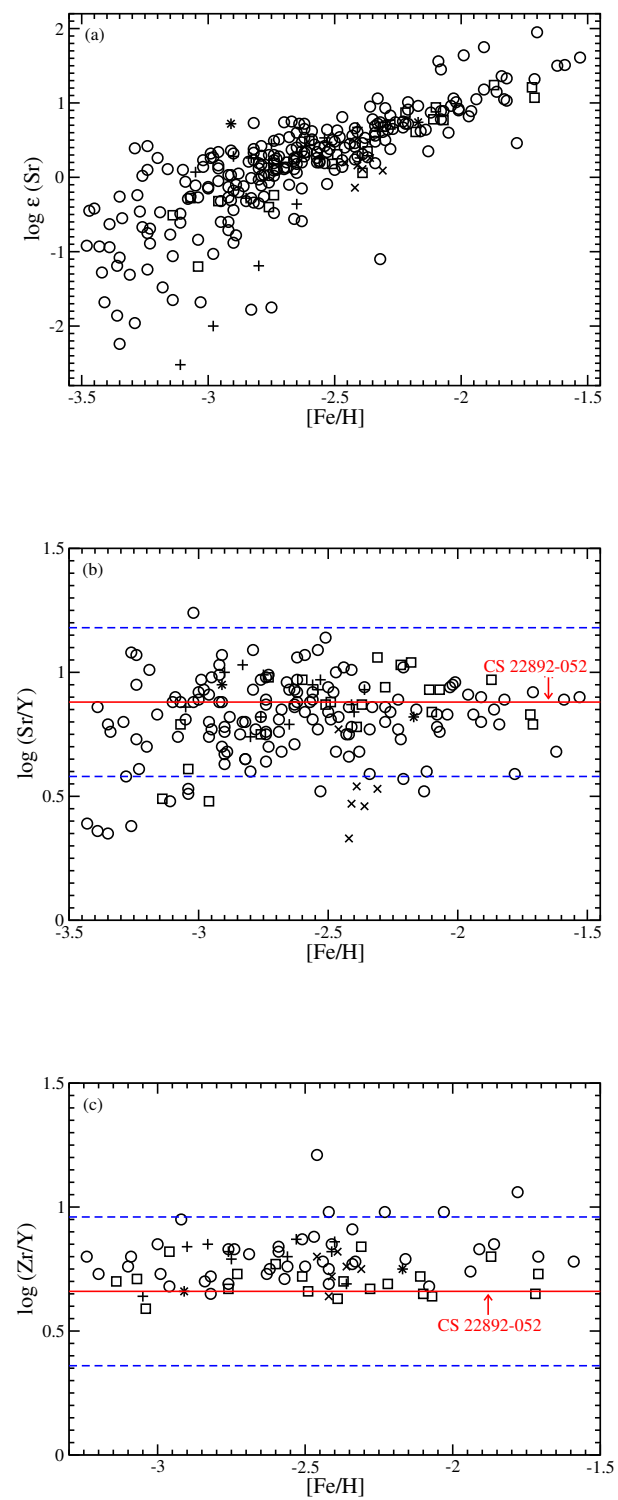

Fig. 4. (a) Data on $\log \epsilon(\mathrm{Sr})$ versus $[\mathrm{Fe} / \mathrm{H}]$ for metal-poor stars [squares: Johnson \& Bolte (2002); pluses: Aoki et al. (2005); circles: Barklem et al. (2005); crosses: Otsuki et al. (2006); asterisks: Hill et al. (2002); Ivans et al. (2006)]. There is a large scatter in $\log \epsilon(\mathrm{Sr})$ at any fixed $[\mathrm{Fe} / \mathrm{H}]$ over the wide range $-3.5 \lesssim[\mathrm{Fe} / \mathrm{H}]<-2.5$. This can be accounted for if CPR nuclei are produced in the neutrino-driven wind whether a neutron star is made in Fe core-collapse $\mathrm{SNe}$, or O-Ne-Mg core-collapse $\mathrm{SNe}$, or AIC events. Note that Fe is produced by the first source but not the latter two. (b) Data on $\log (\mathrm{Sr} / \mathrm{Y})$ versus $[\mathrm{Fe} / \mathrm{H}]$ for the same stars shown in (a). Almost all the data lie within 0.3 dex of the value for CS 22892-052, a star with $[\mathrm{Fe} / \mathrm{H}]=-3.1$ but highly enriched in heavy $r$-nuclei (Sneden et al., 2003). (c) Same as (b) but for $\log (\mathrm{Zr} / \mathrm{Y})$. Note the very narrow range for the abundance ratio of this pair of elements. In consideration of observational uncertainties (especially for Sr that is more difficult to measure), it appears from (b) and (c) that CPR nuclei are rather robust products from the neutrino-driven wind in all core-collapse SNe. 
$\sim 1$ Gyr after the big bang due to the timescale required for the evolution of stars of $\sim 2 M_{\odot}$. Likewise, the contributions to the Fe group nuclei from Type Ia SNe (SNe Ia) that are associated with white dwarfs left behind by low-mass stars would also be mostly absent during this epoch. The dominant contributions to the ISM at low metallicities would be from massive stars of the early generations. Study of low-metallicity stars thus should provide a clear opportunity of observing contributions from these sources. Insofar as $r$-sources are associated with core-collapse SNe from massive stars, then contributions from individual (or few) SN events should be discernable. Taking a typical Fe yield of $\sim 0.1 M_{\odot}$ per SN [e.g., Woosley \& Weaver (1995)] and a dilution mass of $\sim 3 \times 10^{4} M_{\odot}$ to mix with the SN ejecta [e.g., Thornton et al. (1998)], we estimate that an ISM of pure big bang debris would be enriched with $[\mathrm{Fe} / \mathrm{H}] \sim-2.6$ by a single Fe-producing $\mathrm{SN}$ using a solar Fe mass fraction of $X_{\odot}(\mathrm{Fe})=1.27 \times 10^{-3}$ (Anders \& Grevesse, 1989). Stars with such low $[\mathrm{Fe} / \mathrm{H}]$ values would provide important clues to whether and how core-collapse SNe are associated with the $r$-process.

As almost all stellar observations yield elemental, but not isotopic abundances, it is necessary to choose diagnostic elements produced solely or predominantly by the $r$-process. Among these elements are Eu, which is commonly observed, and $\mathrm{Re}$, Os, Ir, and $\mathrm{Pt}$, which are rarely observed. In the absence of any contributions from the $s$-process, $\mathrm{Ba}$ (which is more easily measured) is also an important diagnostic. In the case where Th and $\mathrm{U}$ can be measured, this would be clear evidence for contributions from an $r$-process with large $n / s$ (possibly involving fission cycling) as these nuclei can only be made in such a process.

\subsection{Decoupling of the sources for the heavy $r$-nuclei from those for the ele- ments above $\mathrm{N}$ through the Fe group}

As discussed above, the study of metal-poor stars is basic to the understanding of contributions from individual SNe. The H-K survey (Beers et al., 1992) provided an extensive list of metal-poor star candidates. A very important paper by McWilliam et al. (1995) presented high-resolution analyses of 33 extremely metal-deficient stars. This study clearly showed large scatter in $\mathrm{Sr}$ abundance at fixed $[\mathrm{Fe} / \mathrm{H}]$ over a range of $[\mathrm{Fe} / \mathrm{H}]$ (see Fig. 4 4 a for the same result from more recent data) and large scatter in $\mathrm{Ba}$ abundance at $[\mathrm{Fe} / \mathrm{H}] \sim-3$. In an earlier paper (Sneden et al., 1994), the authors of this study reported the discovery of a star, CS 22892-052, with $[\mathrm{Fe} / \mathrm{H}]=-3.1$ but greatly enhanced in heavy $r$-elements. They showed that the abundance pattern of heavy $r$ elements in this star is indistinguishable from the corresponding part of the solar $r$-pattern. As this star possessed an abundance ratio of heavy $r$-element to Fe in excess of 40 times the solar value, McWilliam et al. (1995) concluded 


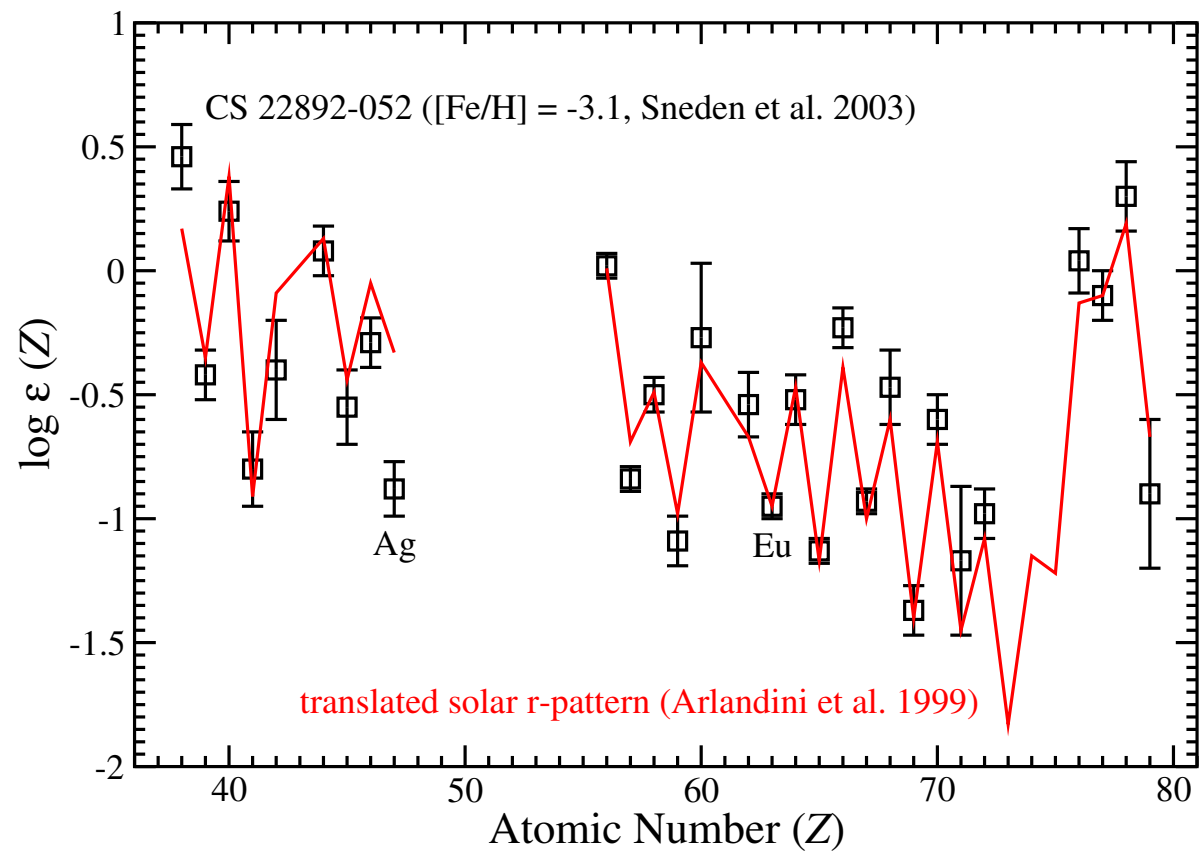

Fig. 5. Data (squares) on abundances of CPR elements and heavy $r$-elements in CS 22892-052 (Sneden et al., 2003). The curves give the overall solar " $r$ "-pattern (shown in Fig. 1 $\mathrm{b}$ ) translated to pass through the data on Eu. Note that the heavy $r$-elements $(Z \geq 56)$ in CS 22892-052 closely follow the corresponding part of the solar $r$-pattern. There is also gross accord for the CPR elements at lower atomic numbers but with clear discrepancies, especially for Ag.

that "the heavy elements are not produced in the same quantities by all type II SN" and that "the SN largely responsible for CS 22892-052 material must be an uncommon occurrence." The possibility discussed below that the production of heavy $r$-nuclei is completely separate from that of the Fe group was not considered. Later Th was also detected in CS 22892-052 (Sneden et al., 1996). Further work (Sneden et al., 2000, 2003) then showed that there was a clear deficiency in Ag relative to the overall solar " $r$ "-pattern (see Fig. 5). As discussed in Section 3, Ag is one of the CPR elements produced by the $\alpha$-process and is not of proper $r$-process origin.

The discovery of a star, CS 31082-001, with $[\mathrm{Fe} / \mathrm{H}]=-2.9$ but greatly enriched in heavy $r$-elements including both Th and $\mathrm{U}$ (both radioactive elements but with very different lifetimes) was reported by Cayrel et al. (2001). The abundance ratio $(\mathrm{Th} / \mathrm{H})$ in this star is $\approx 1 / 12$ the solar value but the ratio $(\mathrm{Fe} / \mathrm{H})$ is only $\approx 1 / 800$ the solar value. The data on this star (Hill et al., 2002 ) are shown as asterisks in Figure 6 with the heavy $r$-elements displayed in the region to the right of the vertical dotted line in Figure 6b. The solid 

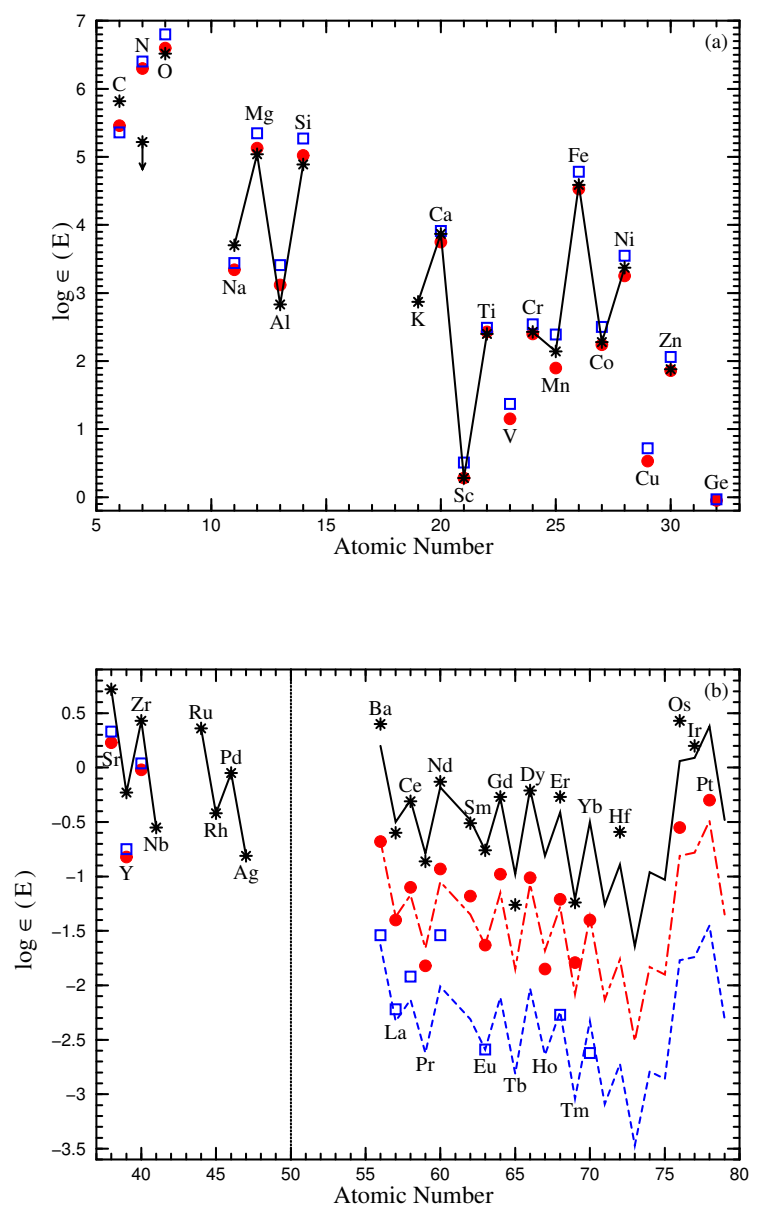

Fig. 6. Data on the elements from C to Pt in CS 31082-001 [asterisks (Hill et al., 2002)], HD 115444 (filled circles), and HD 122563 [squares (Westin et al., 2000)] with $[\mathrm{Fe} / \mathrm{H}]=-2.9,-2.99$, and -2.74 , respectively. (a) The $\log \epsilon$ values for the elements from C to Ge. The data on CS 31082-001 are connected by solid line segments as a guide. Missing segments mean incomplete data. The downward arrow at the asterisk for $\mathrm{N}$ indicates an upper limit. Note that the available abundances for the elements from $\mathrm{O}$ to Ge are almost indistinguishable for the three stars. (b) The $\log \epsilon$ values for the elements from $\mathrm{Sr}$ to $\mathrm{Pt}$. The data on the CPR elements are shown in the region to the left of the vertical dotted line, with those for CS 31082-001 again connected by solid line segments as a guide. There is a rather small range $(\sim 0.5$ dex $)$ in the abundances of the CPR elements for the three stars. In the region to the right of the vertical dotted line, the data on the heavy $r$-elements are compared with the solid, dot-dashed, and dashed curves, which are the solar " $r$ "-pattern translated to pass through the Eu data for CS 31082-001, HD 115444, and HD 122563, respectively. Note the general agreement between the data and these curves. There is a range of $\sim 2$ dex in the abundances of the heavy $r$-elements. Combined with the almost identical abundances of the elements from $\mathrm{O}$ to Ge for the three stars, this strongly suggests that the sources for the heavy $r$-nuclei and those for the elements from $\mathrm{O}$ to Ge are completely decoupled (Qian \& Wasserburg, 2002, 2003). 

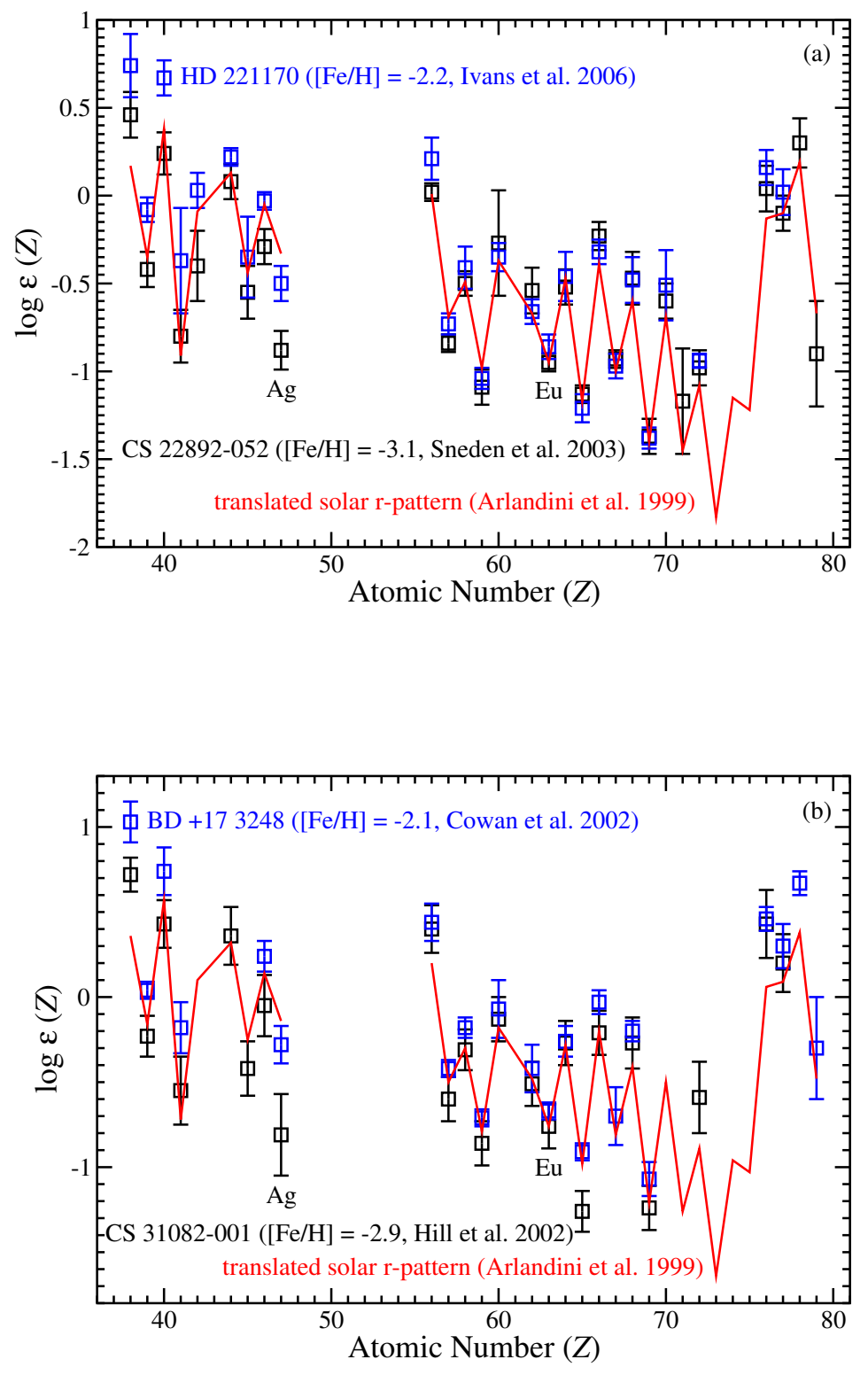

Fig. 7. Comparison of abundances in two pairs of metal-poor stars. (a) CS 22892-052 with $[\mathrm{Fe} / \mathrm{H}]=-3.1$ [black squares (Sneden et al., 2003)] and HD 221170 with $[\mathrm{Fe} / \mathrm{H}]=-2.2$ [blue squares (Ivans et al., 2006)]. (b) CS 31082-001 with $[\mathrm{Fe} / \mathrm{H}]=-2.9$ [black squares (Hill et al., 2002)] and $\mathrm{BD}+17^{\circ} 3248$ with $[\mathrm{Fe} / \mathrm{H}]=-2.1$ [blue squares (Cowan et al., 2002)]. The solid curves in (a) and (b) give the solar " $r$ "-pattern translated to pass through the Eu data for CS 22892-052 and CS 31082-001, respectively. Note that each pair of stars have nearly identical abundances of heavy $r$-elements closely following the solar $r$-pattern but the $(\mathrm{Fe} / \mathrm{H})$ values differ by a factor of 8 and 6 for the pair in (a) and (b), respectively. There are also some differences in the abundances of the CPR elements, especially Ag, for each pair of stars. 
curve in this region represents the solar $r$-pattern translated to pass through the Eu data. It can be seen that there is general agreement between the data on the heavy $r$-elements and the solar $r$-pattern. Studies by several groups provide further examples of stars with a wide range in the abundances of heavy $r$-elements that again approximately follow the solar $r$-pattern. The data on two such stars, HD 115444 with $[\mathrm{Fe} / \mathrm{H}]=-2.99$ and HD 122563 with $[\mathrm{Fe} / \mathrm{H}]=-2.74$ (Westin et al. 2000), are shown as filled circles and open squares, respectively, in Figure 6. The three stars shown in this figure have almost identical abundances of the elements from $\mathrm{O}$ to Ge including Fe (see Fig. 6a), but their heavy $r$-elements differ in abundance by a factor of $\sim 100$ (see Fig. 6b). This led us to conclude that the production of heavy $r$-nuclei is completely separate from the production of the elements of the Fe group and those of intermediate mass above C and N (Qian \& Wasserburg, 2002, 2003).

In addition, we may compare some stars with the same enrichment in heavy $r$-nuclei but with different $[\mathrm{Fe} / \mathrm{H}]$. Figure 7 a shows the data on CS 22892-052 with $[\mathrm{Fe} / \mathrm{H}]=-3.1$ [black squares (Sneden et al., 2003)] and the recent results on HD 221170 with $[\mathrm{Fe} / \mathrm{H}]=-2.2$ [blue squares (Ivans et al., 2006)]. It can be seen that the abundances from $\mathrm{Ba}(Z=56)$ and above are indistinguishable although there is a factor of 8 difference in $(\mathrm{Fe} / \mathrm{H})$ for these two stars. There are some differences in the abundances of the CPR elements, in particular Ag. A similar comparison can be seen in Figure 7b for two other stars, CS 31082-001 [black squares (Hill et al., 2002)] and BD $+17^{\circ} 3248$ [blue squares (Cowan et al., 2002)], which also have nearly the same abundances of heavy $r$-elements but very different $(\mathrm{Fe} / \mathrm{H})$ (by a factor of 6 ) with some differences in the abundances of the CPR elements.

All of the results presented above demonstrate clearly that the heavy $r$-nuclei are not produced in conjunction with the Fe group elements. Further, we note again that the abundances for all the elements from $\mathrm{O}$ to $\mathrm{Ge}$ appear to be almost identical for the three stars with very different enrichments of heavy $r$-elements shown in Figure 6. This suggests that the heavy $r$-nuclei cannot be produced by massive stars of $>11 M_{\odot}$, which result in Fe core-collapse SNe and are sources for the elements from $\mathrm{O}$ to Ge [see the review by Woosley et al. (2002) and Section 4.4.

\subsection{Regularity and variability of the yield pattern of heavy r-nuclei}

It appears that the heavy $r$-elements $\mathrm{Ba}$ and above exhibit an abundance pattern close to the corresponding part of the solar $r$-pattern. This was recognized in all of the observational studies cited above. Two stars discussed above, CS 22892-052 and CS 31082-001, have [Fe/H] $\sim-3$ but extremely high enrichments of heavy $r$-elements $\left[(\mathrm{Eu} / \mathrm{H}) \sim(1 / 30-1 / 20)(\mathrm{Eu} / \mathrm{H})_{\odot}\right]$. These stars 
must represent contributions from single $r$-process sources. However, there is no observational basis for believing that there is a single universal yield pattern even for the heavy $r$-nuclei. In fact, observations show that CS 22892-052 and CS 31082-001 have $\log (\mathrm{Th} / \mathrm{Eu})=-0.62$ (Sneden et al., 2003) and -0.22 (Hill et al., 2002), respectively. This difference of 0.4 dex is much larger than the observational error of $\sim 0.05$ dex (Hill et al., 2002). Further, it cannot be attributed to the possible difference in age between the two stars as ${ }^{232} \mathrm{Th}$ (the only long-lived isotope of Th) has an extremely long lifetime of $\tau_{232}=20.3 \mathrm{Gyr}$

- even if the two stars were born 13.5 Gyr (age of the universe) apart, this would only give a difference of 0.3 dex in $\log (\mathrm{Th} / \mathrm{Eu})$. Therefore, there is good reason to believe that the yields of Th and $\mathrm{U}$ relative to those of heavy $r$ elements (e.g., Eu) below $A \sim 195$ should be variable. This variation renders calculations of stellar ages from $(\mathrm{Th} / \mathrm{Eu})$ rather uncertain. Even a $30 \%$ shift in the yield ratio of Th to Eu would give a shift of 6.1 Gyr in age due to the long lifetime of ${ }^{232} \mathrm{Th}$.

Further evidence for variations in the yield pattern of heavy $r$-nuclei has been found in $\mathrm{HD} 122563$ with $[\mathrm{Fe} / \mathrm{H}]=-2.77$ (Honda et al., 2006). The data on this star (squares) are compared with the solar " $r$ "-pattern (red curves) translated to pass through the Eu data in Figure 8a. It can be seen that there is approximate accord between the data and the solar $r$-pattern for the heavy $r$-elements $(Z \geq 56)$, but there are also large discrepancies, especially for Ce and $\operatorname{Pr}$ (see also the comparison between the squares and the dashed curve in Fig. 6b). Clearly, it is important for future measurements to determine whether such discrepancies extend to other heavy $r$-elements in HD 122563, particularly Os, Ir, and $\mathrm{Pt}$ in the peak at $A \sim 195$ of the solar $r$-pattern. Discovery of other stars of this kind would help establish the range of variations in the yield pattern of heavy $r$-nuclei.

\subsection{Relationship between heavy $r$-nuclei and CPR elements}

Figure $8 \mathrm{a}$ also shows that the $\mathrm{CPR}$ elements from $\operatorname{Sr}(Z=38)$ to $\mathrm{Ag}$ in HD 122563 clearly lie above the solar " $r$ "-pattern translated to pass through the Eu data and extended into this region. This is in sharp contrast to the cases for CS 22892-052, CS 31082-001, BD +17³248, and HD 221170 shown in Figure 7. Therefore, we must conclude that there is large variation in the production of heavy $r$-nuclei relative to CPR elements for different sources or that CPR elements sometimes may be produced independently of heavy $r$-nuclei.

All the available observations show that high enrichments of heavy $r$-nuclei are always accompanied by enrichments of CPR elements. If some stars were found to be highly enriched in heavy $r$-nuclei but without any significant abundances 

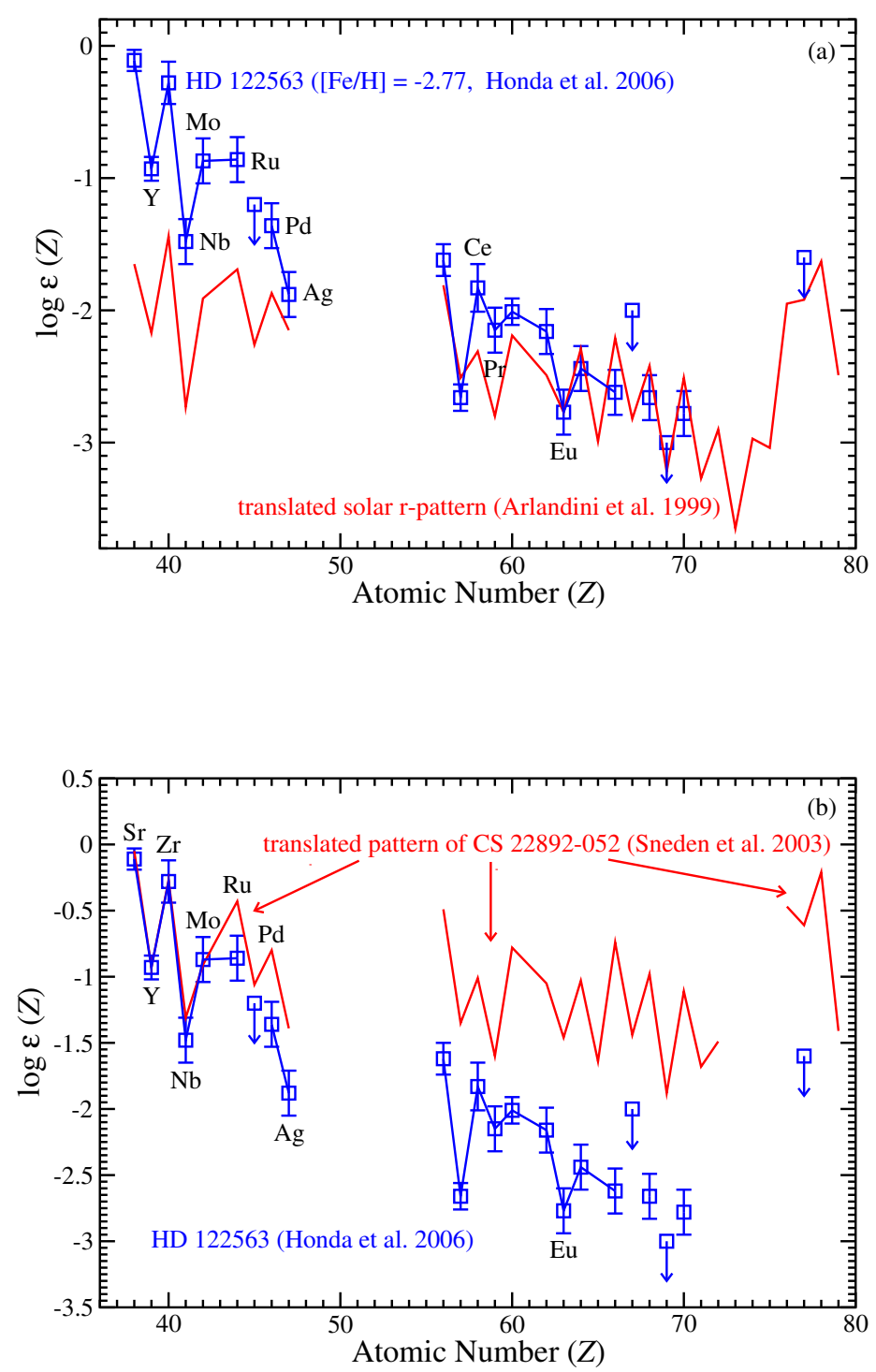

Fig. 8. (a) Data on HD 122563 [squares (Honda et al., 2006)] compared with the solar " $r$ "-pattern translated to pass through the Eu data (red curves). The squares are connected with blue line segments as a guide. Squares with downward arrows indicate upper limits. The abundance pattern of heavy $r$-elements $[\mathrm{Ba}(Z=56)$ and above] in HD 122563 shown in (a) is similar to the corresponding part of the solar " $r$ "-pattern but with substantial differences, especially for Ce and Pr. Note that $(\mathrm{Ba} / \mathrm{Eu})_{\mathrm{HD} 122563} \approx(\mathrm{Ba} / \mathrm{Eu})_{\odot, r}$. There are also gross differences between the data and the translated solar " $r$ "-pattern for the CPR elements. Specifically, HD 122563 has much larger proportions of CPR elements relative to heavy $r$-nuclei as compared to the solar " $r$ "-pattern. (b) Comparison of the data on HD 122563 [squares (Honda et al. 2006)] with those on CS 22892-052 [red curves (Sneden et al., 2003)] normalized to the same $\log \epsilon(\mathrm{Y})$ as for HD 122563. The large difference (by a factor of $\sim 20$ ) in the production of heavy $r$-nuclei relative to CPR elements shown in (b) suggests that only some sources for CPR elements can also produce heavy $r$-nuclei. 
of CPR elements, this would bring serious doubt to any core-collapse SN model for the production of heavy $r$-nuclei. In the absence of such observations, we may assume that a roughly fixed amount of CPR elements is produced in the neutrino-driven wind whenever a neutron star is formed in a core-collapse SN as discussed in Section 3 (see also Section 6.2). Under this assumption, we can normalize the data on different stars to the same abundance of a typical CPR element (e.g., Y) to see the variation in the production of heavy $r$-nuclei relative to CPR elements. The data on CS 22892-052 [red curves (Sneden et al., 2003)] normalized to the same $\log \epsilon(\mathrm{Y})$ as for HD 122563 are compared with those on the latter star [squares (Honda et al., 2006)] in Figure 8b. The large difference (by a factor of $\sim 20$ ) in the production of heavy $r$-nuclei [Ba $(Z=56)$ and above] relative to CPR elements shown in this figure (see also Fig. 6b) can be accounted for if only some core-collapse SNe can produce the heavy $r$-nuclei along with the CPR elements but others can only produce the CPR elements without any heavy $r$-nuclei. This is then fully consistent with the conclusion reached in Section 4.1 that heavy $r$-nuclei cannot be produced by Fe core-collapse SNe from progenitors of $>11 M_{\odot}$, which are sources for the elements above $\mathrm{N}$ through the Fe group.

\subsection{Implications for sources of heavy $r$-nuclei}

Whether the elements of the Fe group and those of intermediate mass above $\mathrm{N}$ can be produced by a core-collapse $\mathrm{SN}$ is closely related to the pre-SN structure of the progenitor. Figure 9 compares the composition structure at the onset of core collapse for two stars with initial masses of 25 (top panel) and $11 M_{\odot}$ (bottom panel), respectively. Both stars had solar metallicity initially and were evolved from the main sequence with mass loss (Woosley et al., 2002). It can be seen that while both stars have developed an Fe core, the $25 M_{\odot}$ star has a clear Si shell and an extensive $\mathrm{O}$ shell but the $11 M_{\odot}$ star does not. The transition between these two kinds of composition structure occurs at $\sim 12 M_{\odot}$ [e.g., Woosley \& Weaver (1995)]. The elements from Si to the Fe group are produced by explosive burning as the SN shock propagates through the $\mathrm{Si}$ and $\mathrm{O}$ shells. (Note that the original Fe nuclei in the inner core become free nucleons at nuclear density and those in the outer core are dissociated by the shock. Therefore, none of the Fe nuclei in the initial core are ejected in the SN.) It is considered that Fe core-collapse SNe from progenitors of $\lesssim 25 M_{\odot}$ eject most of their nucleosynthetic products and leave behind neutron stars [e.g., Woosley et al. (2002)]. Thus, we expect that the products of explosive nucleosynthesis are ejected along with the elements produced by pre-SN hydrostatic burning in the outer shells for Fe core-collapse SNe from progenitors of $\sim 12-25 M_{\odot}$. In other words, a host of nuclei from $\mathrm{C}$ to the Fe group would be ejected from such core-collapse SNe. In contrast, Fe corecollapse SNe from progenitors of $\sim 11 M_{\odot}$ eject very little of the elements of 

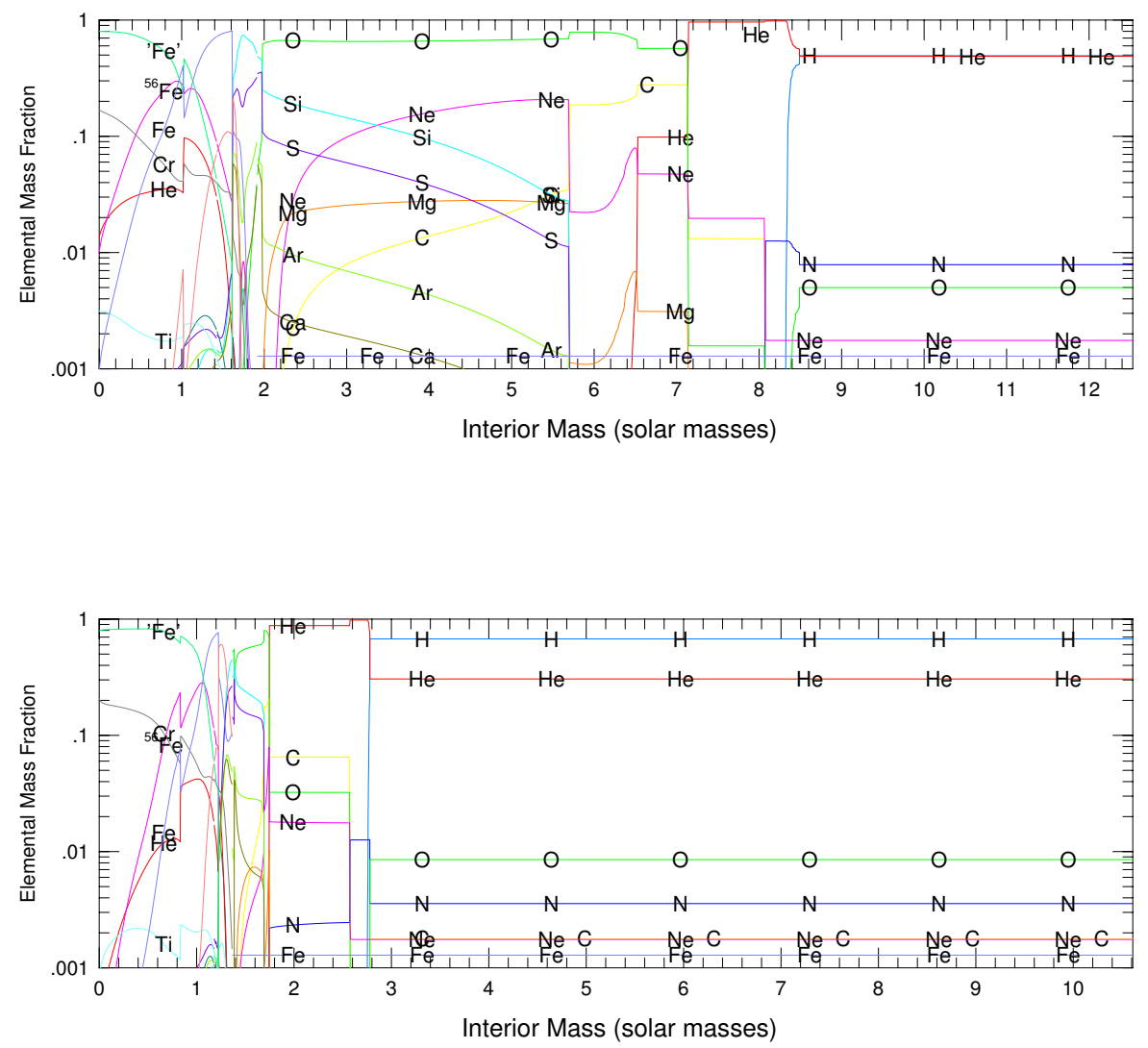

Fig. 9. Composition structure at the onset of core collapse in terms of the mass fractions of various elements as functions of the mass coordinate for two stars with initial masses of 25 (top panel) and $11 M_{\odot}$ (bottom panel), respectively. Both stars had solar metallicity initially and were evolved from the main sequence with mass loss (Woosley et al. 2002). While both stars have developed an Fe core, the $25 M_{\odot}$ star has a clear Si shell and an extensive $\mathrm{O}$ shell but the $11 M_{\odot}$ star only has a $\mathrm{He}$ shell between the Fe core and the $\mathrm{H}$ envelope.

the Fe group or those of intermediate mass above $\mathrm{N}$ as these stars only have a He shell between the Fe core and the $\mathrm{H}$ envelope (note that the Fe outside the core shown in Fig. 9 is due to the assumed initial metallicity of the star).

The evolution of stars of $\sim 8-10 M_{\odot}$ is more complicated [see reviews by Nomoto \& Hashimoto (1988); Woosley et al. (2002); Herwig (2005)]. Nomoto (1984, 1987) studied the evolution of He cores of $2.2,2.4$, and $2.6 M_{\odot}$ corresponding to stars of $8.8,9.6$, and $10.4 M_{\odot}$ and showed that these stars develop $\mathrm{O}-\mathrm{Ne}-\mathrm{Mg}$ cores. The composition structure prior to core collapse for He cores of 2.4 (case 2.4) and 2.6 $M_{\odot}$ (case 2.6) is shown in the top and bottom panels of Figure 10, respectively. Note that case 2.4 has a simple O-Ne-Mg core but case 2.6 has a more evolved outer core outside the inner O-Ne-Mg core. 

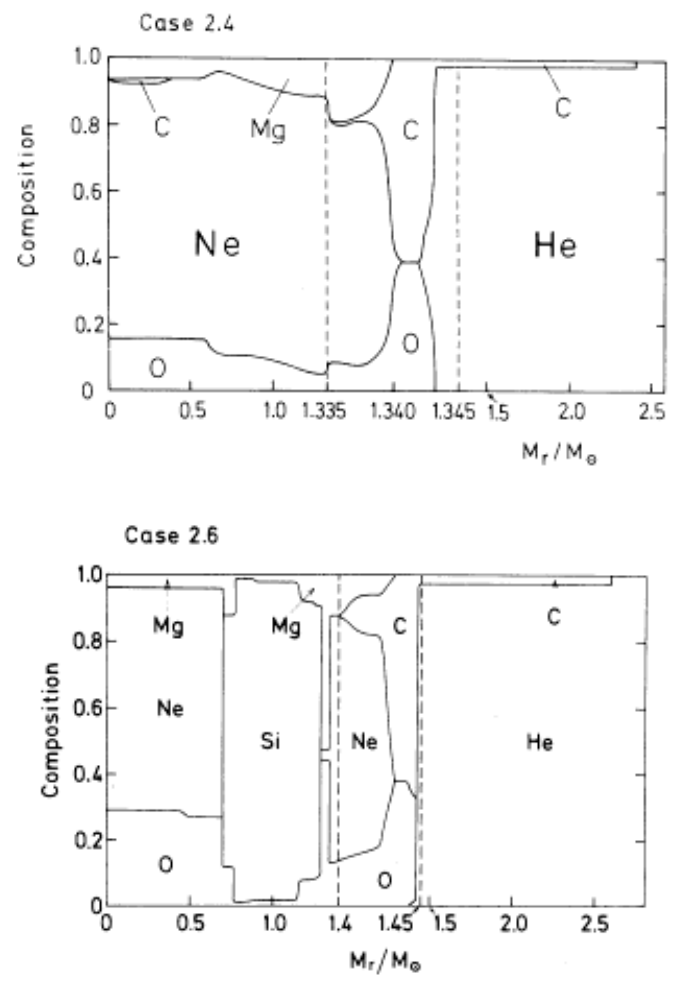

Fig. 10. Composition structure prior to core collapse for He cores of 2.4 (case 2.4) and $2.6 M_{\odot}$ (case 2.6) corresponding to stars of 9.6 and $10.4 M_{\odot}($ Nomoto, 1984). At a given mass coordinate, the vertical spacing between the boundaries of the region marked with an element gives the mass fraction of this element. Note that case 2.4 has a simple $\mathrm{O}-\mathrm{Ne}-\mathrm{Mg}$ core but case 2.6 has a more evolved outer core outside the inner O-Ne-Mg core. In both cases, there is only a very thin C-O shell between the core and the He shell. The mass coordinate in this region (between vertical dashed lines) is greatly magnified.

In both cases, there is only a very thin C-O shell between the core and the He shell. While the further evolution of case 2.6 is rather uncertain, Nomoto 1984, 1987) concluded that He cores of $2-2.5 M_{\odot}$ corresponding to progenitors of $8-10 M_{\odot}$ result in O-Ne-Mg core-collapse SNe that leave neutron stars but produce very little of the elements of the Fe group or those of intermediate mass above $\mathrm{N}$.

An intermediate-mass star of $\lesssim 8 M_{\odot}$ would become a C-O or O-Ne-Mg white dwarf after passing through the AGB phase and losing its envelope. If such a star is in a binary system with a low-mass companion, the white dwarf produced by this star may accrete sufficient material from the companion and collapse into a neutron star (Nomoto \& Kondo, 1991). Accretion is limited by the supply from the companion. As the white dwarf must exceed the Chandrasekhar mass of $\approx 1.4 M_{\odot}$ in order to collapse, this AIC scenario may work 
only for the more massive white dwarfs produced by stars of $\sim 5-8 M_{\odot}$. The neutron star produced by an AIC event would have a neutrino-driven wind where CPR elements are made. However, with any overlying envelope being very small, such an event would not produce the elements above $\mathrm{N}$ through the Fe group.

From the above discussion and the considerations in the preceding subsections, we are left with a limited number of scenarios for the production of the heavy $r$-nuclei. Insofar as this production is directly or indirectly related to the neutrino-driven wind from a nascent neutron star, there are only three possibilities: Fe core-collapse SNe from progenitors of $\sim 11 M_{\odot}, \mathrm{O}-\mathrm{Ne}-\mathrm{Mg}$ core-collapse SNe from progenitors of $\sim 8-10 M_{\odot}$, and AIC of white dwarfs left behind by stars of $\sim 5-8 M_{\odot}$ in binary systems. None of these sources produce the elements above $\mathrm{N}$ through the Fe group, but all of them produce CPR elements in the neutrino-driven wind. We will refer to the first two possibilities collectively as low-mass core-collapse SNe. The nucleosynthetic signatures of the AIC scenario will be discussed in more detail in Section 4.5. Other workers have proposed a low-mass core-collapse SN source for the $r$-process based on considerations of Galactic evolution of Eu with Fe [e.g., Mathews et al. (1992); Ishimaru \& Wanajo (1999)] or parametric models of the $r$-process [e.g., Wanajo et al. (2003)]. These considerations and models are not related to the critical and definitive observations and arguments laid out in the preceding subsections. The arguments outlined here [see also Qian \& Wasserburg [2002, 2003)] are based on the observed decoupling of the production of heavy $r$-nuclei from that of the elements above $\mathrm{N}$ through the Fe group as well as the relationship between heavy $r$-nuclei and CPR elements.

One might argue that Fe core-collapse $\mathrm{SNe}$ with progenitors of $>25 M_{\odot}$ may not eject the elements above $\mathrm{N}$ through the Fe group due to "fallback" [e.g., Woosley \& Weaver (1995)], and therefore could be a source for the heavy $r$ nuclei. There are two problems with this argument. First, unlike the elements from Si to the Fe group, which are produced by explosive burning in the inner shells and therefore most susceptible to fallback for progenitors of $>$ $25 M_{\odot}$, the elements $\mathrm{O}, \mathrm{Na}, \mathrm{Mg}$, and $\mathrm{Al}$ are produced by hydrostatic burning in the outer shells during pre-SN evolution and will not be as easily eliminated by fallback [e.g., Woosley \& Weaver (1995)]. Further, the heavy $r$-nuclei are usually considered to be made in the inner most part of an SN. Were they made in Fe core-collapse SNe with progenitors of $>25 M_{\odot}$, it is very difficult to see how they can survive the fallback that prevents the ejection of those elements produced in the outer regions.

It follows that low-mass core-collapse SNe and AIC events are the only possible sources for heavy $r$-nuclei. In reaching this conclusion, we have assumed that stars of $\sim 8-11 M_{\odot}$ would lead to low-mass core-collapse SNe that do not produce the elements of the Fe group or those of intermediate mass above 
N. Investigations by several groups indicate that the evolution of stars in this narrow mass interval depends on the initial metallicity and is especially sensitive to the treatment of convection and convective overshoot (Woosley et al., 2002). In particular, the exact mass range resulting in O-Ne-Mg corecollapse SNe is rather uncertain. For example, Ritossa et al. (1999) found that for solar metallicity, only stars of $11 M_{\odot}$ lead to O-Ne-Mg core-collapse SNe while those of 9,10 , and $10.5 M_{\odot}$ become O-Ne-Mg white dwarfs after ejecting their H envelope [see García-Berro \& Iben (1994); Ritossa et al. (1996); GarcíaBerro et al. (1997); Iben et al. (1997)]. This is in conflict with the results shown in Figures 9 and 10 . Because stars of $\sim 8-11 M_{\odot}$ play such important roles in understanding the sources for heavy $r$-nuclei, it is highly desirable that further work be pursued to follow the evolution of these stars starting from the main sequence and considering the effects of the initial metallicity and mass loss.

\subsection{Occurrence of $s$ and $r$-processes in binary systems}

The AIC scenario discussed in the preceding subsection may have a rather complicated history of mass transfer between the primary intermediate-mass star of $\sim 5-8 M_{\odot}$ and its low-mass companion. The companion may accrete material from the primary star during the red giant branch (RGB) and AGB stages of the latter, thus acquiring typical products of RGB and AGB evolution, in particular the $s$-process nuclei ( $s$-nuclei). The primary star would eventually eject its envelope and become a white dwarf. If this white dwarf were then to accrete sufficient material from the expanded low-mass companion, it could collapse into a neutron star. While no elements above $\mathrm{N}$ through the Fe group would be produced in this AIC event, CPR elements would be made in the neutrino-driven wind from the neutron star. If heavy $r$-nuclei could also be made in this event, then the low-mass companion would acquire these nuclei as well as the CPR elements through contamination of its surface by the ejecta containing such products. In this scenario, a low-mass star in a binary system may become highly enriched first with AGB products including $\mathrm{C}, \mathrm{N}$, and $s$-nuclei and then with CPR elements and heavy $r$-nuclei Qian \& Wasserburg, 2003). Extensive reviews of nucleosynthesis in AGB stars have been given by Busso et al. (1999) and Herwig (2005).

The above scenario was motivated by the important discovery of a dwarf star, HE 2148-1247, which has $[\mathrm{Fe} / \mathrm{H}]=-2.3$ but is highly enriched in neutroncapture elements (Cohen et al. 2003). The observed abundances in this star are shown as squares in Figure 11 . Specifically, this star has nearly solar values of $(\mathrm{Ba} / \mathrm{H}),(\mathrm{La} / \mathrm{H}),(\mathrm{Ce} / \mathrm{H}),(\mathrm{Pr} / \mathrm{H})$, and $(\mathrm{Nd} / \mathrm{H})$, half the solar value of $(\mathrm{Eu} / \mathrm{H})$, and 6.6 times the solar value of $(\mathrm{Pb} / \mathrm{H})$. In addition, its $(\mathrm{C} / \mathrm{H})$ and $(\mathrm{N} / \mathrm{H})$ are 0.4 and 0.2 times the corresponding solar values, respectively, with ${ }^{12} \mathrm{C} /{ }^{13} \mathrm{C} \sim 10(\sim 9$ times less than the solar ratio). Being a dwarf star, 

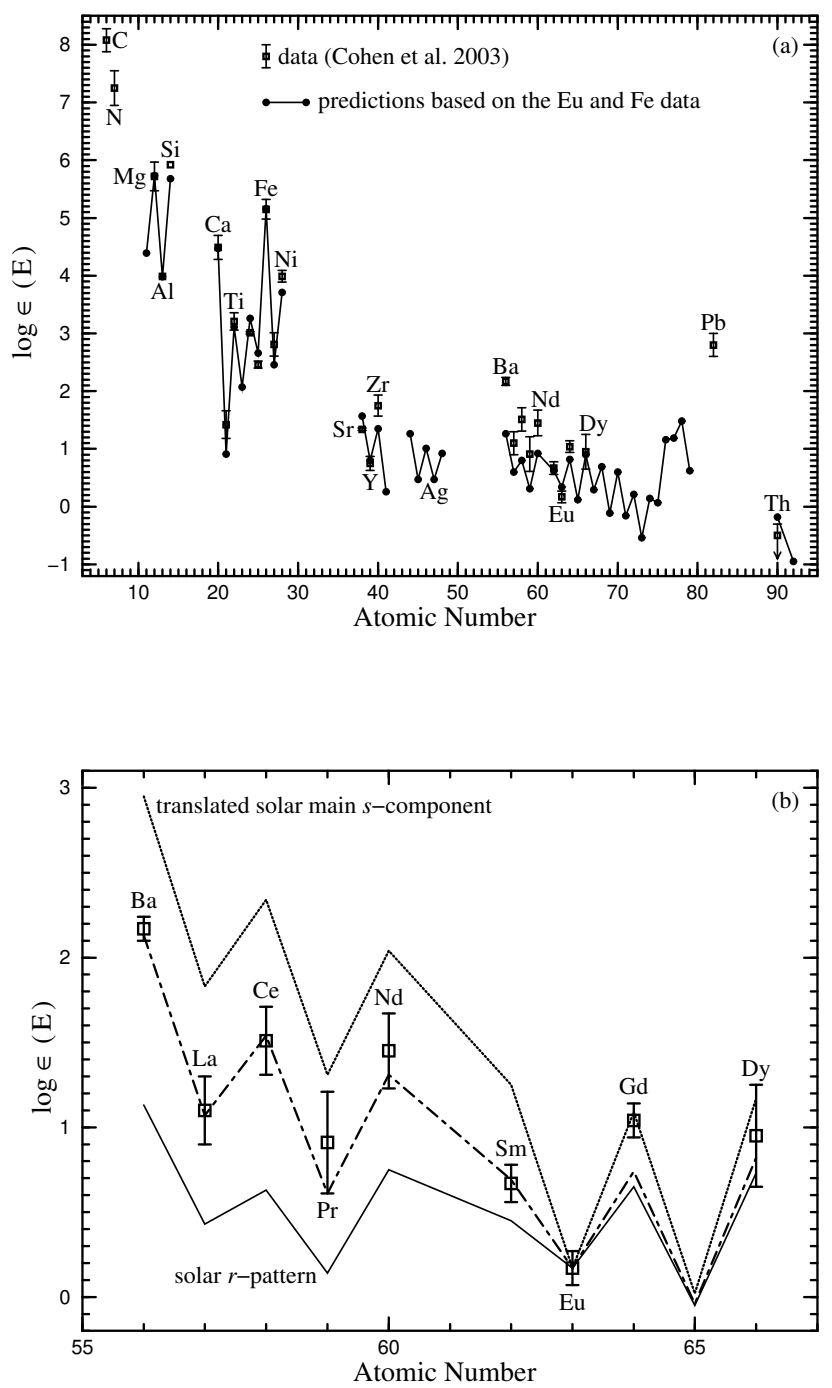

Fig. 11. Data (squares) on the " $s+r$ " star HE 2148-1247 with $[\mathrm{Fe} / \mathrm{H}]=-2.3$ discovered by Cohen et al. (2003). (a) All data from C to Th are shown. The filled circles connected with solid line segments are predictions based on the original "LEGO-block" model of Qian \& Wasserburg (2001, 2002) using only the Eu and Fe abundances for the star (see Section 5). This model does not treat the $s$-process contributions. Note that $\mathrm{Ba}$ is far above the prediction and $\mathrm{Pb}$ stands very high. (b) The data on the elements from Ba to Dy are compared with the solar $r$-pattern (thin solid curve) and the solar main s-component (dotted curve), both of which are translated to pass through the Eu data. It is clear that the data must represent a mixture of the solar $r$-pattern and the solar main $s$-component. The dot-dashed curve shows such a mixture with $86 \%$ of the Eu contributed by the $r$-process and the corresponding fractional $r$-process contributions to the other elements. This mixture describes the data very well except for Gd. 
HE 2148-1247 could not have produced the observed high enrichments in C, $\mathrm{N}$, and neutron-capture elements by itself. These enrichments must represent the result of mass transfer from a more massive and rapidly evolving binary companion that had gone through the RGB and AGB stages. Indeed, this star is observed to be a radial velocity variable and is most likely a member of a long-period binary system (Cohen et al., 2003). The clear enhancement of $\mathrm{Ba}$ relative to $\mathrm{Eu}$ and gross enhancement of $\mathrm{Pb}$ demonstrate that the surface material of this star had undergone extensive s-processing. On the other hand, the $\mathrm{Ba} / \mathrm{Eu}$ ratio is much smaller than those typically obtained in $s$-process models [e.g., Van Eck et al. (2001)], thus requiring significant contributions from the $r$-process. Except for $\mathrm{Gd}$ and $\mathrm{Pb}$, the data on other neutron-capture elements in HE 2148-1247 can be fitted very well by a mixture of the solar main $s$-component and the solar $r$-pattern with $86 \%$ of the Eu contributed by the $r$-process QQian \& Wasserburg (2003), see Fig. 11b]. Metal-poor stars with both high $s$ and $r$-enrichments appear to be quite common in binary systems. Shortly after the discovery of HE 2148-1247 by Cohen et al. (2003), similar results on another star, CS $29497-030$, with $[\mathrm{Fe} / \mathrm{H}]=-2.16$ were reported by Sneden et al. (2003). The latter star is also in a binary system with evidence of mass transfer.

The data on $\epsilon(\mathrm{Ba})$ versus $\epsilon(\mathrm{Eu})$ for a sample of metal-poor stars with $[\mathrm{Fe} / \mathrm{H}]<$ -2 were shown in Figure 13 of Cohen et al. (2003). This figure is reproduced here as Figure 12. The dot-dashed line represents the solar Ba/Eu ratio with the letter "S" on this line indicating the position of the Sun. The solid line represents the $\mathrm{Ba} / \mathrm{Eu}$ ratio for the solar $r$-pattern and the dashed line for the solar main $s$-component. HE 2148-1247 is shown as the large filled circle in Figure 12. It can be clearly seen that this low-metallicity star has near solar $\mathrm{Ba}$ and $\mathrm{Eu}$ abundances with a $\mathrm{Ba} / \mathrm{Eu}$ ratio slightly above that in the Sun. It can also be seen that there are a number of other stars like HE 2148-1247 exhibiting both high $s$ and $r$-process enrichments. A more recent compilation of such stars, all of which appear to be greatly enhanced in C, can be found in Jonsell et al. (2006). In addition to reporting the new results on HE 03383945 , these authors provided a comprehensive summary of both observations on similar stars in the literature [including those of Cohen et al. (2003) and Sneden et al. (2003)] and theoretical interpretations of these results.

In the cases where both $s$ and $r$-process additions have been made to a lowmass star in a binary system, one may question whether the $r$-nuclei were already present in the material from which this star and its binary companion were formed. The viewpoint that the $r$-process material was already present in the ISM from which these stars were formed has been pursued by several groups [e.g., Ivans et al. (2005); Aoki et al. (2006)]. We first note that in some of the stars with both $s$ and $r$-process enhancements, the level of $r$-enrichment is extremely high (see Fig. 12). For example, HE 2148-1247 has half the solar value of $(\mathrm{Eu} / \mathrm{H})$. If we assume that over the Galactic history of $\sim 10 \mathrm{Gyr}$, 


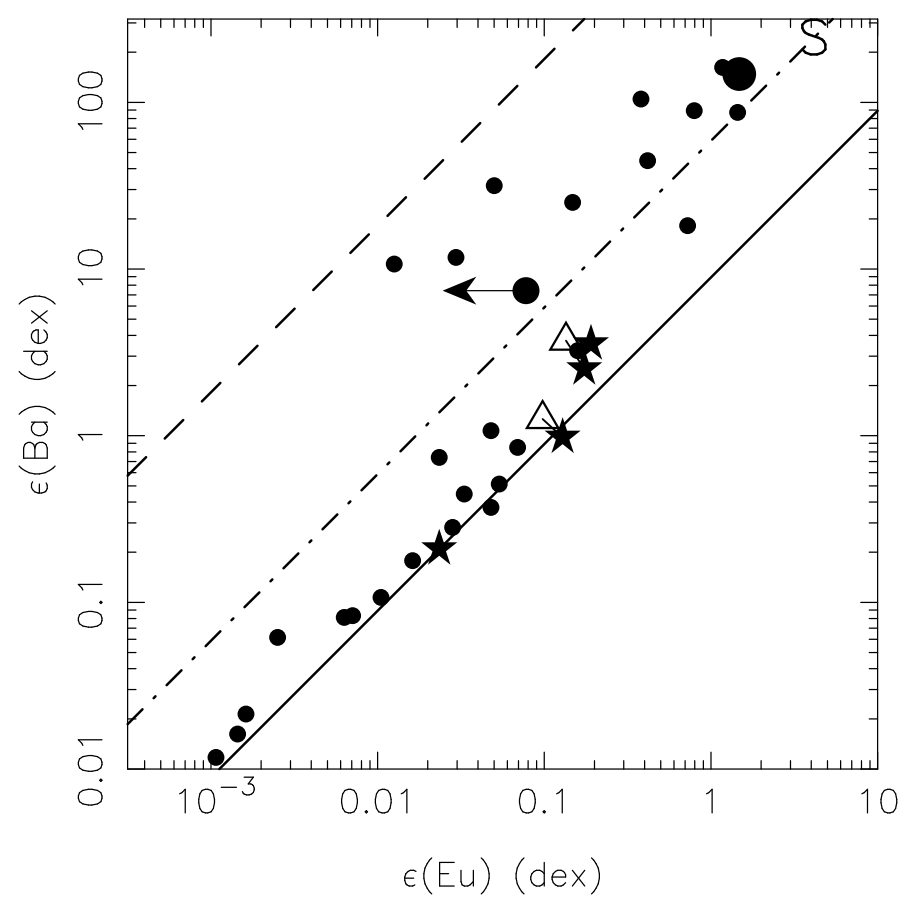

Fig. 12. Data on $\epsilon(\mathrm{Ba})$ and $\epsilon(\mathrm{Eu})$ for a sample of metal-poor stars with $[\mathrm{Fe} / \mathrm{H}]<-2$ compiled by Cohen et al. (2003). See that paper for the sources of the data. The dot-dashed line represents the solar $\mathrm{Ba} / \mathrm{Eu}$ ratio with the letter " $\mathrm{S}$ " on this line indicating the position of the Sun. The solid line represents the $\mathrm{Ba} / \mathrm{Eu}$ ratio for the solar $r$-pattern and the dashed line for the solar main $s$-component. HE 2148-1247 is shown as the large filled circle. The filled circle with the arrow represents a star with only an upper limit on Eu. It can be seen that there are a number of other stars like HE 2148-1247 exhibiting both high $s$ and $r$-process enrichments.

$\sim 10^{8}$ core-collapse SNe provided a solar $(\mathrm{Eu} / \mathrm{H})$ to a total of $\sim 10^{10} M_{\odot}$ of gas, then to provide $(\mathrm{Eu} / \mathrm{H})$ at the solar level by a single SN would require that the ejecta from this SN be mixed with only $\sim 100 M_{\odot}$ of ISM. This mixing mass is much smaller than the typical value of $\sim 3 \times 10^{4} M_{\odot}$ for SN ejecta [e.g., Thornton et al. (1998)]. It is also known that the " $s+r$ " combination is rather common in metal-poor stars in binary systems but extremely high $r$-enrichments are rare among single metal-poor stars [e.g., Jonsell et al. (2006)]. Thus the above approach requires an arbitrary intimacy between a heavily $r$-enriched ISM and the formation of a binary system. We find it difficult to accept the notion that metal-poor binary systems were somehow preferentially selected to inherit very high $r$-enrichments from a precursor ISM or unrelated but intimate source. We consider the observational data to most strongly support the point of view that the " $s+r$ " mixtures in low-metallicity stars represent mass transfer involving both $s$ and $r$-process products between these stars and their binary companions and that such binary systems were formed with initial compositions typical of the ISM. Note that in our pre- 
ferred scenario, the $s$-processing occurs during the AGB phase of the primary star and precedes the $r$-processing proposed to occur during the later AIC event. Thus, in contrast to the scenario assuming that the binary system was formed from heavily $r$-enriched ISM, the heavy $r$-nuclei are not exposed to $s$-processing in our scenario.

We again note that the ejecta from an $r$-process event is diluted by a large amount of ISM so that stars formed from an ISM enriched by a small number of events would be unlikely to exhibit high $r$-enrichments. Observations targeted toward metal-poor stars with extreme $r$-enhancements would not be sampling the dominant population of stars formed from such an ISM. Instead, such observations would automatically be biased toward stars with extreme $s+r$ enhancements in binary systems. However, the results from these observations do not imply that the $r$-process only occurs in binary systems as they do not represent $r$-enrichment of the general ISM.

With regard to the calculations of $s$-processing in low-metallicity stars, there are several issues that require attention. First, calculations of the $s$-process in these stars have typically assumed that the neutron source is from a ${ }^{13} \mathrm{C}$ pocket. It is not at all evident that this is applicable in the circumstances where AIC is involved. There the stars in which the $s$-process occurs must be of $\sim 5-8 M_{\odot}$ and may have considerably different characteristics from the stars of $\sim 1-3 M_{\odot}$ usually associated with the main $s$-process. In particular, at the more elevated temperatures in the intermediate-mass stars, the reaction ${ }^{22} \mathrm{Ne}(\alpha, n)^{25} \mathrm{Mg}$ will play a significant role. Calculations that just use the neutrons from this source may give significantly different $s$-yield patterns from those produced with the standard ${ }^{13} \mathrm{C}$ pocket. Aoki et al. (2006) recently reported for the first time the detection of Os and Ir in CS 31062-050, a star with $[\mathrm{Fe} / \mathrm{H}]=-2.3$ but with high $s+r$ enrichments [see also Johnson \& Bolte (2004)]. The elements Os and Ir are predominantly made by the $r$-process and their abundances in CS 31062-050 relative to the Eu enhancement found by Johnson \& Bolte (2004) are compatible with the solar $r$-pattern. In accounting for the observed abundances of Eu, Os, and Ir, Aoki et al. (2006) assumed that these $r$-enrichments were inherited by the star from the ISM (see discussion in the two preceding paragraphs). The highly enhanced $\mathrm{Pb}$ in CS 31062-050 again clearly shows the presence of an s-component produced by a low-metallicity AGB star as predicted by Gallino et al. (1998). However, as noted by Aoki et al. (2006) and seen in Figure 13, the $s$-process model proposed to explain the $\mathrm{Pb}$ clearly fails to explain the $\mathrm{Ba}$ and some of the rare-earth elements (e.g., Er to Hf). It does not appear that the observations can be described using a standard $s$-process model. Studies of the $s$-process in low-metallicity intermediate-mass stars of $\sim 5-8 M_{\odot}$ must be undertaken in order to better understand the problem at hand.

The question of whether a higher-mass star of e.g., $\sim 10 M_{\odot}$ could produce 


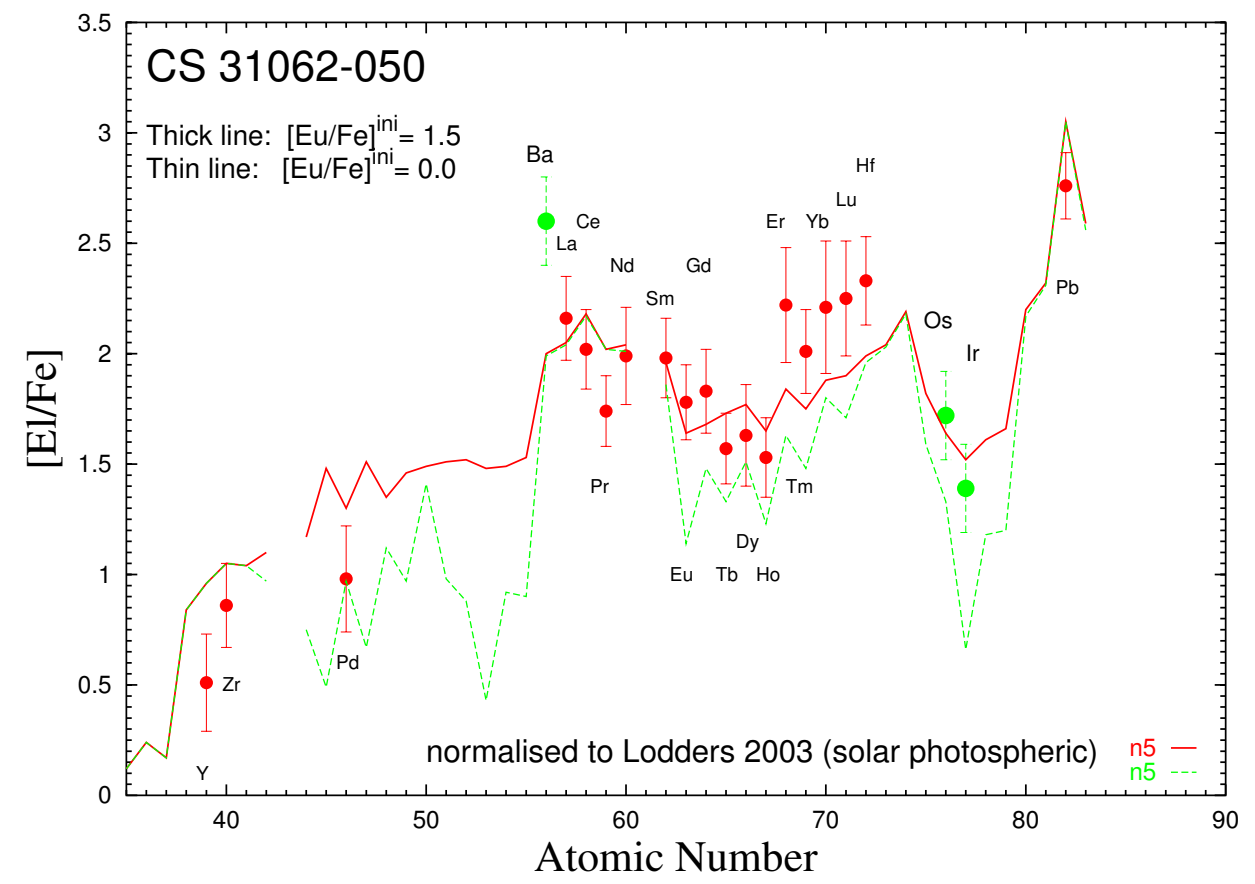

Fig. 13. Data [red filled circles: Johnson \& Bolte (2004); green filled circles: Aoki et al. (2006)] on the elemental abundances relative to $\mathrm{Fe}$ in terms of $[\mathrm{El} / \mathrm{Fe}] \equiv \log (\mathrm{El} / \mathrm{Fe})-\log (\mathrm{El} / \mathrm{Fe}) \odot$ for CS 31062-050 with $[\mathrm{Fe} / \mathrm{H}]=-2.3$. As shown by Johnson \& Bolte (2004), the very high abundances of $\mathrm{Ba}$ and $\mathrm{Pb}$ as well as $\mathrm{Eu}$ require a mixture of $s$ and $r$-process contributions. The new measurements by Aoki et al. (2006) on Os and Ir, both predominantly made by the $r$-process, show that the abundances of these two elements relative to $\mathrm{Eu}$ are compatible with the solar $r$-pattern. The solid red curve gives the results from a theoretical model that calculated the $s$-process yields using the standard ${ }^{13} \mathrm{C}$ pocket and assuming an initial $r$-process inventory corresponding to $[\mathrm{Eu} / \mathrm{Fe}]^{\text {ini }}=1.5$ inherited from the ISM. The green dashed line is for a similar model with $[\mathrm{Eu} / \mathrm{Fe}]^{\text {ini }}=0$. It can be seen that a general overall agreement between the models and data was obtained for some elements using $\mathrm{Pb}$ as the key guide. However, there is no particular agreement for many other elements including $\mathrm{Ba}$ as noted by Aoki et al. (2006). The problem of full modelling of " $s+r$ " mixtures is a matter for ongoing studies.

the $s$-nuclei and transfer them to a low-mass binary companion also remains to be addressed. Such a binary system could lead to the same general results as for the AIC scenario outlined above. With a primary star of $\sim 10 M_{\odot}$, the $r$-nuclei may be produced by the O-Ne-Mg core-collapse SN from this star (assuming a neutron source). This was preferred by some workers [e.g., Wanajo et al. (2006)] because it does not rely on the transfer of material from the low-mass companion to induce the collapse as in the AIC scenario. As noted in Section 4.4, the evolution of stars of $\sim 8-11 M_{\odot}$ is rather complex. This 
mass range is bounded from below by the onset of O-Ne-Mg core formation and from above by the onset of Fe core formation. Neither boundaries have been clearly determined [see e.g., the reviews by Woosley et al. (2002) and Herwig (2005)]. Whether stars in the above mass range leave an O-Ne-Mg white dwarf or undergo $\mathrm{O}-\mathrm{Ne}-\mathrm{Mg}$ core collapse to produce a neutron star also requires further investigation. A complete knowledge of the full stellar evolution for stars of $\sim 8-11 M_{\odot}$ is urgently needed. This has been mentioned by Herwig (2005), who referred to these as "super-AGB" stars. A new report on the evolution of such stars by Poelarends et al. (2006) is in preparation.

We also note that some studies of the detailed evolution of single intermediatemass stars have been made [e.g., Karakas \& Lattanzio (2004)], but none of them carried out $s$-process calculations. The same is true of the higher masses of $\sim 10 M_{\odot}$. For further progress to be made, it is now evident that detailed calculations for the evolution of binary systems with a primary member of $\sim 5$ $11 M_{\odot}$ and a low-mass companion, including $s$-processing and various episodes of mass transfer, are necessary in order to tell whether mass transfer of all resulting elements $(\mathrm{C}, \mathrm{N}$, and $s$-nuclei) can plausibly explain the observational data at hand and the increasing quantity of such data.

\subsection{Where has the r-process gone?}

We summarize the observations on metal-poor stars in terms of the following rather strict rules on the $r$-process:

(1) The production of heavy $r$-nuclei with $A>130$ is fully decoupled from that of Fe and all the other elements between $\mathrm{N}$ and Ge.

(2) The production of CPR nuclei with $A \sim 90-110$ is not tightly coupled to that of heavy $r$-nuclei. (All the available observations show that while metalpoor stars highly enriched with heavy $r$-nuclei always have enrichments of CPR nuclei at a comparable level, there are also stars enriched with CPR nuclei but having very low abundances of heavy $r$-nuclei.)

The meteoritic data on ${ }^{129} \mathrm{I}$ and ${ }^{182} \mathrm{Hf}$ discussed in Section 1 give the third rule:

(3) The production of heavy $r$-nuclei including ${ }^{182} \mathrm{Hf}$ is decoupled from that of light $r$-nuclei with $A \lesssim 130$ including ${ }^{129} \mathrm{I}$.

To infer the possible sites for the $r$-process, we combine the above rules with the following aspects of stellar evolution and nucleosynthesis discussed in Sections $3,4.4$, and 4.5 . 
(a) Stars of $\sim 12-25 M_{\odot}$ result in Fe core-collapse SNe, which produce a neutron star of $\approx 1.4-2 M_{\odot}$ and are sources for the elements between $\mathrm{N}$ and Ge.

(b) A neutron star of $\approx 1.4 M_{\odot}$ is produced in: Fe core-collapse SNe from progenitors of $\sim 11 M_{\odot} ; \mathrm{O}-\mathrm{Ne}-\mathrm{Mg}$ core-collapse $\mathrm{SNe}$ from progenitors of $\sim 8$ $10 M_{\odot}$; and $\mathrm{AIC}$ of white dwarfs from progenitors of $\sim 5-8 M_{\odot}$ in binary systems. None of these core-collapse SNe produce the elements between $\mathrm{N}$ and Ge.

(c) The nascent neutron star produced by any of the core-collapse SNe in (a) and (b) has a neutrino-driven wind, which is a natural source for CPR nuclei.

Table 1 summarizes aspects (a)-(c) and shows the logical alternatives from considering these and rules (1)-(3). If we assume that there is an adequate neutron source directly or indirectly related to the neutrino-driven wind from all neutron stars by some unknown mechanism, then all the core-collapse SNe listed above could provide both light and heavy $r$-nuclei. However, in consideration of the strict decoupling of heavy $r$-nuclei from Fe [rule (1)], Fe core-collapse SNe from progenitors of $\sim 12-25 M_{\odot}$ are excluded as a source for heavy $r$-nuclei. If we also consider the meteoritic data [rule (3)] and assume that ${ }^{182} \mathrm{Hf}$ and other heavy $r$-nuclei are produced by low-mass SNe from progenitors of $\sim 8-11 M_{\odot}$ and AIC events, then these sources cannot be the source for the light $r$-nuclei. This leaves Fe core-collapse SNe from progenitors of $\sim 12-25 M_{\odot}$ as a possible source for the light $r$-nuclei. As all the core-collapse SNe listed above are sources for CPR nuclei but only low-mass SNe and AIC events could provide heavy $r$-nuclei, rule (2) on the relationship between these two groups of nuclei is also satisfied.

Regarding the heavy $r$-nuclei, we again note the remarkable fact that their abundance pattern is rather constant. It follows that there must be a rather fixed nuclear processing scheme for producing them. A plausible mechanism is fission cycling. The meteoritic data require that ${ }^{129} \mathrm{I}$ not be produced together with ${ }^{182} \mathrm{Hf}$ and other heavy $r$-nuclei. This sets a lower bound of $A \sim 130$ on the nuclei produced by fission cycling if it is responsible for the yield pattern of heavy $r$-nuclei. However, we have no direct observational evidence on the nuclei with $A \sim 130$ from metal-poor stars that might elucidate the matter. If fission cycling is operative, it must permit some variability in the yield pattern over $130<A \lesssim 195$ (as shown by some data sets, see Figs. 6 and 8 a) and in the yield of Th relative to Eu (as shown by the data on CS 22892-052 and CS 31082-001, see discussion in Section 4.2). Fission cycling would be rather unlikely if some stars were observed to have high enrichments in nuclei with $130<A \lesssim 195$ but very low Th abundances.

In any case, the neutron source for producing the heavy $r$-nuclei is not known 
Table 1

Characteristics of possible sources for CPR and $r$-process nuclei

\begin{tabular}{|c|c|c|c|}
\hline Progenitor & $\mathrm{AIC}$ & $8-11 M_{\odot}$ & $12-25 M_{\odot}$ \\
\hline Neutron star mass & $\approx 1.4 M_{\odot}$ & $\approx 1.4 M_{\odot}$ & $\approx 1.4-2 M_{\odot}$ \\
\hline Fe source? & no & no & yes \\
\hline \multicolumn{4}{|c|}{ considering neutrino-driven winds } \\
\hline CPR source? & yes & yes & yes \\
\hline \multicolumn{4}{|c|}{ assuming neutron sources } \\
\hline$A \lesssim 130$ source? & yes & yes & yes \\
\hline$A>130$ source? & yes & yes & yes \\
\hline \multicolumn{4}{|c|}{ considering rule $(1)$ on decoupling of $A>130$ from Fe } \\
\hline$A>130$ source? & yes & yes & no \\
\hline \multicolumn{4}{|c|}{ considering rule (1) and rule (3) on decoupling of ${ }^{182} \mathrm{Hf}$ from ${ }^{129}$} \\
\hline$A \lesssim 130$ source? & no & no & yes \\
\hline
\end{tabular}

and does not appear to be in the neutrino-driven wind. If the wind is not the source of the neutrons, then some other mechanism must exist in the same stellar environment but independent of the wind. This undetermined mechanism appears to be restricted to stars of $\lesssim 11 M_{\odot}$. We do not know the answer to this puzzle and would be delighted if anyone knew!!

There is a little children's song:

Oh where, oh where has my little dog gone,

Oh where, oh where can he be?

With his ears cut short and his tail cut long,

Oh where, oh where can he be?

For the $r$-process that we know exists, we propose the following ditty:

Oh where, oh where has my $r$-process gone,

Oh where, oh where can it be?

With neutrons cut short and "neutrino" winds long,

Oh where, oh where can it be?

We end this long section with a cautionary note. We here have only explored the consequences of the decoupling between Fe and heavy $r$-nuclei. The sharp decoupling of the production of $\mathrm{Fe}$ and other elements between $\mathrm{N}$ and Ge from that of heavy $r$-nuclei is based upon a considerable body of observations on low-metallicity stars. However, such observations would have missed lowmass stars enriched by the following hypothetical scenario. If somehow a low- 
metallicity star of $\sim 11 M_{\odot}$ managed to produce heavy $r$-nuclei and ejected a substantial amount of Fe (e.g., from its original Fe core by some unknown mechanism) at the same time, and if it was in a binary system with a low-mass companion, then the surface of the companion would have been contaminated with large amounts of heavy $r$-nuclei and Fe. If the primary star had also gone through an AGB phase, it would have produced a large amount of $\mathrm{Pb}$ by the $s$-process at low-metallicity. Whether the binary system remains intact or was disrupted, the original low-mass companion would now appear to be highly enriched in heavy $r$-nuclei and $\mathrm{Pb}$ but also be Fe rich. Such stars would not be sampled by the observations directed toward halo stars with low to very low $[\mathrm{Fe} / \mathrm{H}]$ values. An effort to investigate halo stars with high $[\mathrm{Fe} / \mathrm{H}]$ values and very large $\mathrm{Pb}$ enhancements might clarify whether the above cautionary scenario is plausible.

\section{A LEGO-block model of elemental abundances in stars and the ISM at low metallicities}

We have outlined our consideration of the characteristics exhibited by stellar sources of elements in the regime of $[\mathrm{Fe} / \mathrm{H}] \lesssim-1.5$ where only massive stars might contribute to the ISM. This led to a series of rules (or regularities) for the presumed elemental yield patterns of possible prototypical sources. Based on these rules we attempted to use a simple three-component model to explain the abundances in stars and the ISM at low metallicities in general. This "LEGO-block" model has three assumed building blocks:

$H$ - the heavy $r$-nuclei along with some CPR nuclei from a source (low-mass core-collapse SNe and AIC events) producing no elements between N and Ge,

$L$ - the light $r$-nuclei and CPR nuclei from another source (Fe core-collapse SNe from progenitors of $\sim 12-25 M_{\odot}$ ) producing the elements between $\mathrm{N}$ and Ge including Fe,

$P$ - a universal prompt inventory from the first very massive stars (VMSs).

The prompt inventory ( $P$ inventory) in our original papers on the LEGOblock model (Qian \& Wasserburg, 2001, 2002) was motivated by the apparent jump in $\mathrm{Ba}$ and $\mathrm{Eu}$ abundances at $[\mathrm{Fe} / \mathrm{H}] \sim-3$ shown by the data available then, which suggested that in early epochs VMSs prevailed producing Fe and elements of lower atomic numbers but no heavy $r$-nuclei. The element Eu that is almost exclusively of $r$-process origin was used to serve as an index for contributions from the $H$ source. The element Fe was considered to be exclusively produced by the $L$ source at $[\mathrm{Fe} / \mathrm{H}]$ exceeding the value $[\mathrm{Fe} / \mathrm{H}]_{P}$ for the $P$ inventory and used as an index for $L$ contributions. 
A colorful photo showing many Lego blocks.

Fig. 14. Early major stellar sources for the elements in the ISM (and the intergalactic medium) after the initial contributions from the big bang. These are very massive first generation stars (VMSs) and core-collapse SNe [mostly Type II SNe (SNe II)]. There would not have been sufficient time for SNe Ia to occur at the early epochs. Two types of SNe II are considered with SNe II $(H)$ producing heavy $r$-nuclei but no Fe and $\mathrm{SNe} \mathrm{II}(L)$ producing Fe and related elements. Some intermediate-mass stars may also have been involved (e.g., in producing heavy $r$-nuclei from AIC events in binary systems). This simple approach is then used to model stellar abundances for $[\mathrm{Fe} / \mathrm{H}] \lesssim-1.5$.

This approach led to the following rule for the abundance of element E relative to $\mathrm{H}$ for arbitrary stars formed from the ISM (not in binary systems experiencing local $s$-process contamination) at $[\mathrm{Fe} / \mathrm{H}] \lesssim-1.5$ :

$$
\left(\frac{\mathrm{E}}{\mathrm{H}}\right)=\left(\frac{\mathrm{E}}{\mathrm{H}}\right)_{P}+\left(\frac{\mathrm{E}}{\mathrm{Eu}}\right)_{H}\left(\frac{\mathrm{Eu}}{\mathrm{H}}\right)+\left(\frac{\mathrm{E}}{\mathrm{Fe}}\right)_{L}\left[\left(\frac{\mathrm{Fe}}{\mathrm{H}}\right)-\left(\frac{\mathrm{Fe}}{\mathrm{H}}\right)_{P}\right]
$$

This rule was, of course, also applicable to the solar abundances corrected for the $s$-process contributions from AGB stars and the contributions to the Fe group elements from SNe Ia. In particular, the solar $r$-abundance of E was calculated as

$$
\begin{aligned}
\left(\frac{\mathrm{E}}{\mathrm{H}}\right)_{\odot, r} & =\left(\frac{\mathrm{E}}{\mathrm{H}}\right)_{\odot}\left(1-\beta_{\odot, s}\right) \\
& =\left(\frac{\mathrm{E}}{\mathrm{H}}\right)_{P}+\left(\frac{\mathrm{E}}{\mathrm{Eu}}\right)_{H}\left(\frac{\mathrm{Eu}}{\mathrm{H}}\right)_{\odot, r}+\left(\frac{\mathrm{E}}{\mathrm{Fe}}\right)_{L}\left[\left(\frac{\mathrm{Fe}}{\mathrm{H}}\right)_{\odot, L}-\left(\frac{\mathrm{Fe}}{\mathrm{H}}\right)_{P}\right],
\end{aligned}
$$

where $\beta_{\odot, s}$ is the fraction of $\mathrm{E}$ in the Sun produced by the net $s$-process and $(\mathrm{Fe} / \mathrm{H})_{\odot, L}$ is the $L$ contribution to the solar Fe inventory.

It follows that if $(\mathrm{E} / \mathrm{H})_{P},(\mathrm{E} / \mathrm{Eu})_{H}$, and $(\mathrm{E} / \mathrm{Fe})_{L}$ are known for all the elements, then the value of $(\mathrm{E} / \mathrm{H})$ for a metal-poor star is determined by its $(\mathrm{Eu} / \mathrm{H})$ and $(\mathrm{Fe} / \mathrm{H})$. For elements of the Fe group and lower atomic numbers that receive no $H$ contributions, the $(\mathrm{E} / \mathrm{H})_{P}$ values were taken from the data on stars with $[\mathrm{Fe} / \mathrm{H}] \sim-4$ to -3 and scaled to $[\mathrm{Fe} / \mathrm{H}]_{P}=-3$, and the $(\mathrm{E} / \mathrm{Fe})_{L}$ values were calculated from Equation (6) using the data on a star with $[\mathrm{Fe} / \mathrm{H}]=-2$. It turned out that for these elements, the $P$ inventory was almost identical to the $L$-yield pattern, except for small but significant shifts in some of the Fe group elements (Qian \& Wasserburg, 2002). For the heavy $r$-nuclei produced only by the $H$ source, the $(\mathrm{E} / \mathrm{Eu})_{H}$ values were taken to be the same as those for the solar $r$-pattern. For the CPR nuclei, the $(\mathrm{E} / \mathrm{H})_{P}$ and $(\mathrm{E} / \mathrm{Eu})_{H}$ were calculated from Equation (6) using the data on two stars with $[\mathrm{Fe} / \mathrm{H}] \approx-3$ but high enrichments of heavy $r$-nuclei, and the $(\mathrm{E} / \mathrm{Fe})_{L}$ values were calculated from Equation (8) using $(\mathrm{Fe} / \mathrm{H})_{P}=10^{-3}(\mathrm{Fe} / \mathrm{H})_{\odot}$, 
$(\mathrm{Fe} / \mathrm{H})_{\odot, L}=(\mathrm{Fe} / \mathrm{H})_{\odot} / 3$, and the $(\mathrm{E} / \mathrm{H})_{\odot, r}$ values from Arlandini et al. (1999).

Using the $P$ inventory and the $H$ and $L$-yield patterns obtained by the above procedure, we made a direct comparison between calculated and observed abundances for a large number of metal-poor stars (Qian \& Wasserburg, 2001, $2002)$. Here we show in Figure 15 the comparison for the stars with $[\mathrm{Fe} / \mathrm{H}] \lesssim$ -1.5 from a new set of observations (Hill et al., 2002; Johnson \& Bolte, 2002; Aoki et al., 2005; Barklem et al., 2005, Otsuki et al., 2006; Ivans et al., 2006). The results for $\mathrm{Ba}$ as a representative of the heavy $r$-elements are shown in terms of $\Delta \log \epsilon(\mathrm{Ba}) \equiv \log \epsilon_{\mathrm{cal}}(\mathrm{Ba})-\log \epsilon_{\mathrm{obs}}(\mathrm{Ba})$ as a function of $[\mathrm{Fe} / \mathrm{H}]$ in Figure $15 \mathrm{a}$. It can be seen that the difference between the calculated and observed abundances lies within \pm 0.3 dex of zero for most of the stars. There are some clear outliers. The results for $\mathrm{Sr}$ as a typically observed CPR element are shown in Figure 15b. For the CPR elements Nb, Mo, Ru, Rh, Pd, and Ag, there are only limited data available for a relatively small number of stars (Crawford et al., 1998; Hill et al., 2002; Cowan et al., 2002; Johnson \& Bolte, 2002; Honda et al., 2006; Ivans et al., 2006). The results for all these elements are shown in Figure $15 \mathrm{c}$. As in the case of $\mathrm{Ba}$, the agreement between the model and data for the CPR elements is reasonable for most stars but with some extreme outliers. In this version of the LEGO-block model, we have attributed some Ba production to the $L$ source (Qian \& Wasserburg, 2001, 2002).

As shown above, the predictive power for our simple LEGO-block model is quite surprising. This allowed us to win some bottles of wine of varying quality from some observer friends with whom we made wagers (Hill et al. 2002, Christlieb et al., 2004). Further comparison between this model and observations have been made by other workers [e.g., Fulbright et al. (2004); Venn et al. (2004)]. We also note that our original paper on this model (Qian \& Wasserburg, 2001) showed that a significant revision to the solar $s$ and $r$-abundances of Sr, Y, Zr, and Ba as given by Käppeler et al. (1990) and Arlandini et al. (1999) was required in order to obtain good agreement between the model and the data available then. This revision is reasonable as $\beta_{\odot, s} \sim 1$ for these elements and their solar $r$-abundances derived from subtracting the $s$-process contributions are subject to large uncertainties. Some years later a study of Galactic chemical evolution (GCE) by Travaglio et al. (2004) confirmed the need for such a revision and obtained essentially the same quantitative results as ours (but without citation). These authors attributed the required increase in the $r$-process contributions to $\mathrm{Sr}, \mathrm{Y}$, and $\mathrm{Zr}$ to a new type of stellar source. This attribution is, from our point of view, quite unnecessary.

In all of our discussion we have not treated the stars with exceptionally low $[\mathrm{Fe} / \mathrm{H}]$ values of $<-5$ discovered by Christlieb et al. $(2002)$ and Frebel et al. (2005). It is clear that our model does not explain these observations and we have deferred considering them within the framework of our study. These 

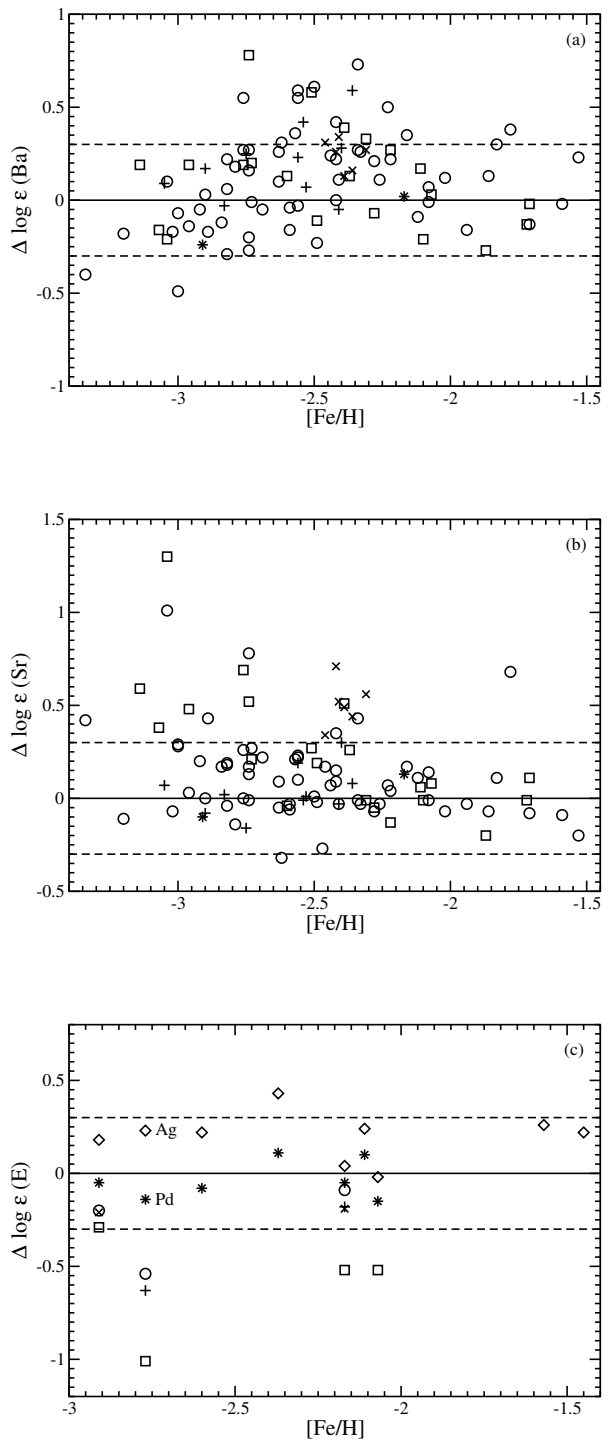

Fig. 15. Comparison of the original "LEGO-block" model with the data for a large sample of stars. (a) The difference between the calculated abundance of Ba and the observed value is shown in terms of $\Delta \log \epsilon(\mathrm{Ba}) \equiv \log \epsilon_{\mathrm{cal}}(\mathrm{Ba})-\log \epsilon_{\mathrm{obs}}(\mathrm{Ba})$ as a function of $[\mathrm{Fe} / \mathrm{H}]$. The calculation uses only the observed $\mathrm{Eu}$ and $\mathrm{Fe}$ abundances. The symbols represent the following data sets: Johnson \& Bolte (2002) (squares), Aoki et al. (2005) (pluses), Barklem et al. (2005) (circles), Otsuki et al. (2006) (crosses), Hill et al. (2002) and Ivans et al. (2006) (asterisks). (b) Same as (a) but for the CPR element Sr. (c) Comparison between the model and data on the other CPR elements are shown. The limited data available are taken from Crawford et al. (1998); Hill et al. (2002); Cowan et al. (2002); Johnson \& Bolte (2002); Honda et al. (2006); Ivans et al. (2006). Only in (c) the symbols represent the CPR elements: $\mathrm{Nb}$ (squares), Mo (pluses), Ru (circles), Rh (crosses), $\mathrm{Pd}$ (asterisks), and $\mathrm{Ag}$ (diamonds). It can be seen from these examples that the elemental abundances in a metal-poor star are often well estimated from the model using only the Eu and Fe abundances to identify the contributions from different sources to the star. However, there are some serious discrepancies. In particular, the attribution of some Ba production to the $L$ source causes the large differences for some stars shown in (a). This attribution is eliminated in the upgraded model (see Section 6.1 and Fig. 16). 
exceptionally metal-poor stars certainly are objects of intense theoretical and observational interest and require understanding. They are exceptions that do not fit into the three-component model outlined here or its successor to be discussed in the following section. Some plausible scenarios for the origins of the abundance patterns in these exceptionally metal-poor stars have been discussed by Iwamoto et al. (2005).

\section{An upgraded LEGO-block model}

\subsection{New yield patterns of $H$ and $L$ sources}

With the intense interest in elemental abundances in low-metallicity stars there is a great increase in high-quality data available as compared with the data sets used by us earlier to develop the original LEGO-block model. From the new results it is found that the $\mathrm{Ba} / \mathrm{Eu}$ ratio is almost constant between $[\mathrm{Fe} / \mathrm{H}] \sim-3.5$ and -2 Hill et al. (2002); Johnson \& Bolte (2002); Aoki et al. (2005); Barklem et al. (2005); Otsuki et al. (2006); Ivans et al. (2006); see Fig. 16]. For the yield patterns used in the original LEGO-block model, it was assumed that significant Ba production occurred in the $L$ source. This is no longer valid and both $\mathrm{Ba}$ and $\mathrm{Eu}$ must be assigned solely to the $H$ source.

Further, in proposing the $P$ inventory VMSs were assumed. As noted in the preceding section, the $P$ inventory for the elements of the Fe group and lower atomic numbers was almost identical to the $L$-yield pattern except for small but significant shifts in some of the Fe group elements [see Fig. 4 of Qian \& Wasserburg (2002)]. The $P$ inventory then mostly affects estimates for the abundances of Sr, $\mathrm{Y}$, and $\mathrm{Zr}$. The $P$ inventory for these elements was calculated from the data on two stars with $[\mathrm{Fe} / \mathrm{H}] \approx-3$ but high enrichments of heavy $r$-nuclei. However, in contrast to the elements of the Fe group and lower atomic numbers, the $P$ inventory of which is in proportion to $(\mathrm{Fe} / \mathrm{H})$ for $[\mathrm{Fe} / \mathrm{H}] \sim-4$ to -3 , the contributions to Sr attributed to the $P$ inventory do not follow this scaling. The general nature of VMS contributions to metal-poor stars also changed as observations of the intergalactic medium (IGM) showed that the cosmic mean abundance of $\mathrm{Si}$ is quite large with $[\mathrm{Si} / \mathrm{H}]_{\mathrm{IGM}}=-2$ accompanied by a rather high value of $[\mathrm{Si} / \mathrm{C}]_{\mathrm{IGM}}=0.77$ (Agurrie et al., 2004). If we assume that VMSs and SN-driven galactic outflows each contributed $50 \%$ of the Si to account for the high $[\mathrm{Si} / \mathrm{C}]_{\mathrm{IGM}}$, then this would have resulted in $[\mathrm{Fe} / \mathrm{H}]_{\mathrm{IGM}} \sim-2.3$ within a few Gyr after the big bang (Qian \& Wasserburg, 2005). This result on the average Fe abundance in the early IGM would not be changed if only galactic outflows contributed the metals. It follows that the low-metallicity stars of concern here, especially those with $[\mathrm{Fe} / \mathrm{H}] \lesssim-2.3$, cannot be representative of the average IGM and the formation of such stars must 


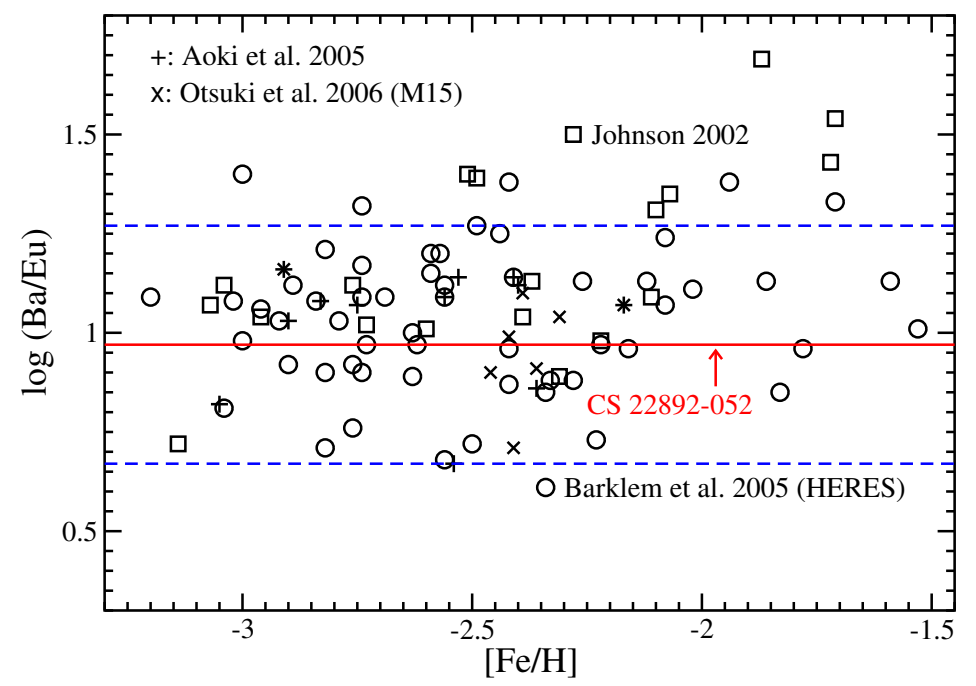

Fig. 16. Data [squares: Johnson \& Bolte (2002); pluses: Aoki et al. (2005); circles: Barklem et al. (2005); crosses: Otsuki et al. (2006); asterisks: Hill et al. (2002); Ivans et al. (2006)] on $\log (\mathrm{Ba} / \mathrm{Eu})$ as a function of $[\mathrm{Fe} / \mathrm{H}]$ for a large sample of stars. Note that the $\log (\mathrm{Ba} / \mathrm{Eu})$ values are clustered around that for the solar $r$-pattern as represented by CS $22892-052$. These values are typically far below $\log (\mathrm{Ba} / \mathrm{Eu})_{\odot}=1.7$. There are clearly some outliers. These results show that $\mathrm{Ba}$ and $\mathrm{Eu}$ in the regime of $[\mathrm{Fe} / \mathrm{H}] \lesssim-1.5$ are governed by $r$-process contributions.

have been fed by the infall of gas from a "metal-poor" IGM into the Galaxy. As a result, we cannot consider a $P$ inventory from VMSs as a component in pursuing the LEGO-block model.

Based on the above discussion, we have changed the model to eliminate the $P$ inventory and only consider the $H$ and $L$ components. In this "upgraded" LEGO-block model, the $H$ source is solely responsible for all the heavy $r$ nuclei (see above discussion on the $\mathrm{Ba} / \mathrm{Eu}$ ratio and Fig. 16). The $(\mathrm{E} / \mathrm{Eu})_{H}$ values for these nuclei are taken from the corresponding part of the solar $r$ pattern calculated by Arlandini et al. (1999). The corresponding $\log (\mathrm{E} / \mathrm{Eu})_{H}$ values are given in Table2, For the CPR nuclei, we obtain their $H$ and $L$-yield patterns from the data on CS 22892-052 (Sneden et al. 2003) and HD 122563 (Honda et al., 2006). These two stars have similar Fe abundances but the former star is highly enriched in Eu while the latter has an Eu abundance lower by a factor of 66 (see Table 3). Clearly, the abundances of CPR nuclei in HD 122563 are dominated by $L$ contributions. So we use the data on this star to calculate the $(\mathrm{E} / \mathrm{Fe})_{L}$ values for these nuclei as

$$
\log \left(\frac{\mathrm{E}}{\mathrm{Fe}}\right)_{L}=\log \epsilon_{\mathrm{HD}}(\mathrm{E})-\log \epsilon_{\mathrm{HD}}(\mathrm{Fe})
$$


Table 2

Yield patterns of heavy $r$-nuclei for the $H$ and $L$ sources in the upgraded LEGOblock model

\begin{tabular}{llllll}
\hline \hline Element & $\log (\mathrm{E} / \mathrm{Eu})_{H}$ & $\log (\mathrm{E} / \mathrm{Fe})_{L}$ & Element & $\log (\mathrm{E} / \mathrm{Eu})_{H}$ & $\log (\mathrm{E} / \mathrm{Fe})_{L}$ \\
\hline $\mathrm{Ba}$ & 0.96 & $-\infty$ & $\mathrm{Tm}$ & -0.45 & $-\infty$ \\
$\mathrm{La}$ & 0.26 & $-\infty$ & $\mathrm{Yb}$ & 0.26 & $-\infty$ \\
$\mathrm{Ce}$ & 0.46 & $-\infty$ & $\mathrm{Lu}$ & -0.50 & $-\infty$ \\
$\mathrm{Pr}$ & -0.03 & $-\infty$ & $\mathrm{Hf}$ & -0.13 & $-\infty$ \\
$\mathrm{Nd}$ & 0.58 & $-\infty$ & $\mathrm{Ta}$ & -0.88 & $-\infty$ \\
$\mathrm{Sm}$ & 0.28 & $-\infty$ & $\mathrm{W}$ & -0.20 & $-\infty$ \\
$\mathrm{Gd}$ & 0.48 & $-\infty$ & $\mathrm{Re}$ & -0.27 & $-\infty$ \\
$\mathrm{Tb}$ & -0.22 & $-\infty$ & $\mathrm{Os}$ & 0.82 & $-\infty$ \\
$\mathrm{Dy}$ & 0.56 & $-\infty$ & $\mathrm{Ir}$ & 0.85 & $-\infty$ \\
$\mathrm{Ho}$ & -0.05 & $-\infty$ & $\mathrm{Pt}$ & 1.14 & $-\infty$ \\
$\mathrm{Er}$ & 0.35 & $-\infty$ & $\mathrm{Au}$ & 0.28 & $-\infty$ \\
\hline
\end{tabular}

where $\log \epsilon_{\mathrm{HD}}(\mathrm{E})$ refers to the data on HD 122563. The calculated $\log (\mathrm{E} / \mathrm{Fe})_{L}$ values are given in the fifth column of Table 3 . Based on these values and the Fe abundance of CS 22892-052, we find that the $L$ contributions to the abundances of CPR nuclei in this star are negligible. We then use the data on CS 22892-052 to calculate the $(\mathrm{E} / \mathrm{Eu})_{H}$ values for these nuclei as

$$
\log \left(\frac{\mathrm{E}}{\mathrm{Eu}}\right)_{H}=\log \epsilon_{\mathrm{CS}}(\mathrm{E})-\log \epsilon_{\mathrm{CS}}(\mathrm{Eu})
$$

where $\log \epsilon_{\mathrm{CS}}(\mathrm{E})$ refers to the data on CS 22892-052. The calculated $\log (\mathrm{E} / \mathrm{Eu})_{H}$ values are given in the fourth column of Table 3.

Using the $H$ and $L$-yield patterns in Table 3, we calculate the abundance of an element $\mathrm{E}$ relative to $\mathrm{H}$ in a star with $[\mathrm{Fe} / \mathrm{H}] \lesssim-1.5$ as

$$
\left(\frac{\mathrm{E}}{\mathrm{H}}\right)=\left(\frac{\mathrm{E}}{\mathrm{Eu}}\right)_{H}\left(\frac{\mathrm{Eu}}{\mathrm{H}}\right)+\left(\frac{\mathrm{E}}{\mathrm{Fe}}\right)_{L}\left(\frac{\mathrm{Fe}}{\mathrm{H}}\right)
$$

The comparison between the calculated abundances of the CPR nuclei and the data is shown in Figure 17 for the same set of stars shown in Figure 15. It can be seen from Figure 17 that the agreement is excellent for $\mathrm{Nb}, \mathrm{Mo}, \mathrm{Ru}$, $\mathrm{Rh}, \mathrm{Pd}$, and $\mathrm{Ag}$ for the limited data available. The agreement for $\mathrm{Sr}$ is also much improved compared with Figure $15 \mathrm{~b}$ although the few outliers still exist. 

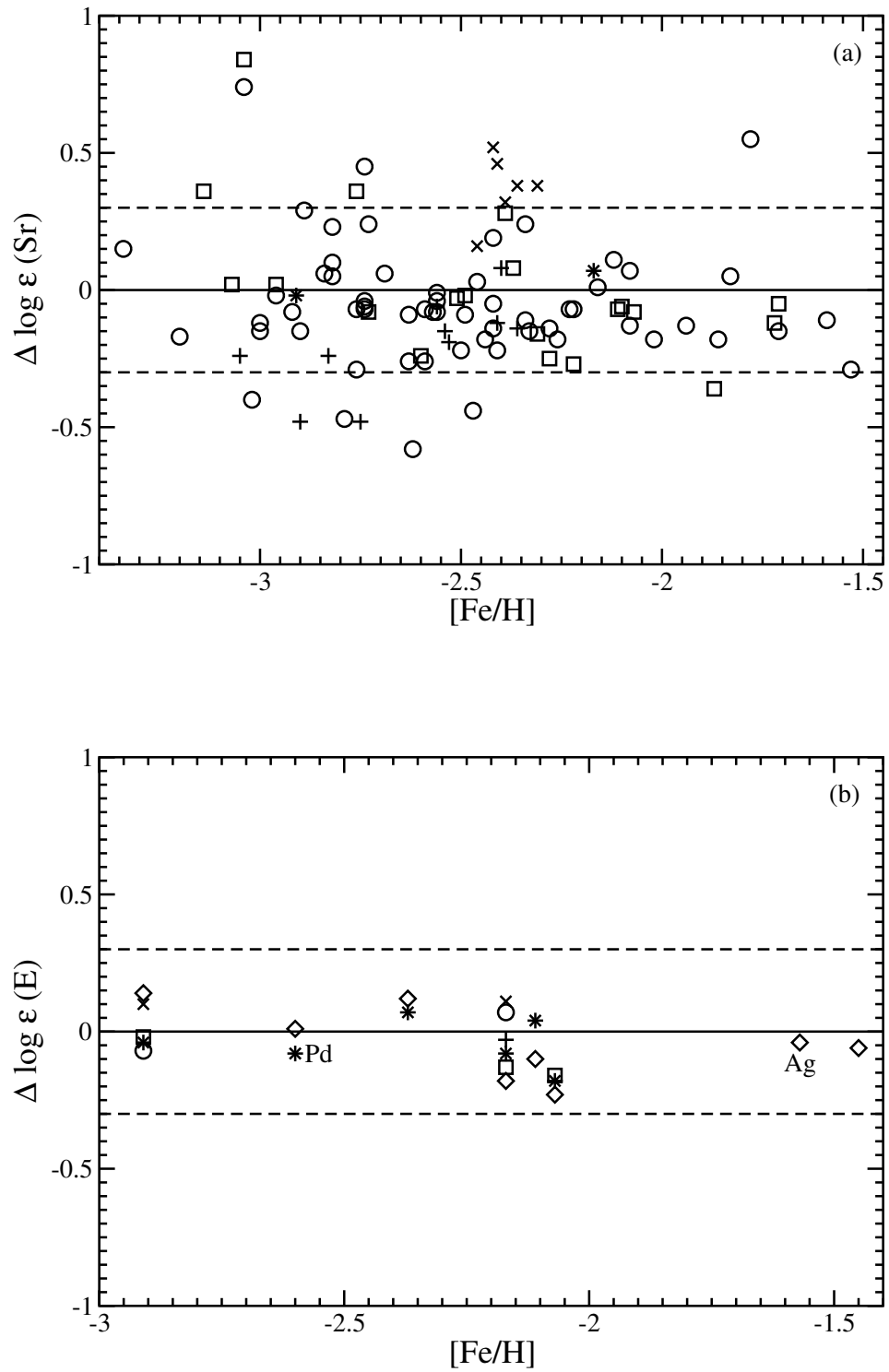

Fig. 17. Comparison of the "upgraded" LEGO-block model with the data for a large sample of stars. (a) The difference between the calculated abundance of the CPR element $\mathrm{Sr}$ and the observed value is shown in terms of $\Delta \log \epsilon(\mathrm{Sr}) \equiv \log \epsilon_{\text {cal }}(\mathrm{Sr})-\log \epsilon_{\mathrm{obs}}(\mathrm{Sr})$ as a function of $[\mathrm{Fe} / \mathrm{H}]$. The calculation uses only the observed $\mathrm{Eu}$ and $\mathrm{Fe}$ abundances. The symbols represent the following data sets: Johnson \& Bolte (2002) (squares), Aoki et al. (2005) (pluses), Barklem et al. (2005) (circles), Otsuki et al. (2006) (crosses), Hill et al. (2002) and Ivans et al. (2006) (asterisks). (b) Comparison between the model and data on the other CPR elements are shown. The limited data available are taken from Crawford et al. (1998); Hill et al. (2002); Cowan et al. (2002); Johnson \& Bolte (2002); Ivans et al. (2006). The symbols represent the CPR elements: Nb (squares), Mo (pluses), $\mathrm{Ru}$ (circles), $\mathrm{Rh}$ (crosses), $\mathrm{Pd}$ (asterisks), and $\mathrm{Ag}$ (diamonds). It can be seen from these examples that the elemental abundances in a metal-poor star are rather well estimated from the model using only the $\mathrm{Eu}$ and $\mathrm{Fe}$ abundances to identify the contributions from different sources to the star. 
Table 3

Input data and yield patterns of CPR nuclei for the $H$ and $L$ sources in the upgraded LEGO-block model

\begin{tabular}{lllll}
\hline \hline & $\log \epsilon_{\mathrm{CS}}(\mathrm{E})$ & $\log \epsilon_{\mathrm{HD}}(\mathrm{E})$ & & \\
Element & $\mathrm{CS} 22892-052$ & $\mathrm{HD} 122563$ & $\log (\mathrm{E} / \mathrm{Eu})_{H}$ & $\log (\mathrm{E} / \mathrm{Fe})_{L}$ \\
\hline $\mathrm{Fe}$ & 4.40 & 4.74 & $-\infty$ & 0 \\
$\mathrm{Eu}$ & -0.95 & -2.77 & 0 & $-\infty$ \\
$\mathrm{Sr}$ & 0.46 & -0.11 & 1.41 & -4.85 \\
$\mathrm{Y}$ & -0.42 & -0.93 & 0.53 & -5.67 \\
$\mathrm{Zr}$ & 0.24 & -0.28 & 1.19 & -5.02 \\
$\mathrm{Nb}$ & -0.80 & -1.48 & 0.15 & -6.22 \\
$\mathrm{Mo}$ & -0.40 & -0.87 & 0.55 & -5.61 \\
$\mathrm{Ru}$ & 0.08 & -0.86 & 1.03 & -5.60 \\
$\mathrm{Rh}$ & -0.55 & $<-1.20$ & 0.40 & $<-5.94$ \\
$\mathrm{Pd}$ & -0.29 & -1.36 & 0.66 & -6.10 \\
$\mathrm{Ag}$ & -0.88 & -1.88 & 0.07 & -6.62 \\
\hline
\end{tabular}

\subsection{Absolute yields of CPR nuclei}

Based on the comparison shown in Figure 17, we consider that the upgraded LEGO-block model with the $H$ and $L$-yield patterns given in Tables 2 and 3 provides a good description of the observations on CPR nuclei. Further, we can use these patterns to estimate the absolute yields of CPR nuclei from each $H$ and $L$ event. As both $H$ and $L$ events involve the formation of neutron stars that have neutrino-driven winds producing CPR nuclei (see Section 3), the yields of these nuclei should be comparable in both cases. We have argued that the $L$ source is Fe core-collapse SNe from progenitors of $\sim 12-25 M_{\odot}$. These SNe have a typical Fe yield of $Y_{L}(\mathrm{Fe}) \sim 0.1 M_{\odot}$ per event [e.g., Woosley \& Weaver (1995)]. With $(\mathrm{Sr} / \mathrm{Fe})_{L}=10^{-4.85}$ (see Table 3), this gives an $L$-yield $Y_{L}(\mathrm{Sr})$ of $\mathrm{Sr}$ as

$$
Y_{L}(\mathrm{Sr})=\left(\frac{\mathrm{Sr}}{\mathrm{Fe}}\right)_{L}\left(\frac{A_{\mathrm{Sr}}}{A_{\mathrm{Fe}}}\right) Y_{L}(\mathrm{Fe}) \sim 2.2 \times 10^{-6} M_{\odot}
$$

where $A_{\mathrm{Sr}} \approx 88$ and $A_{\mathrm{Fe}} \approx 56$ are the mass numbers of $\mathrm{Sr}$ and Fe, respectively. We have also argued that the $H$ source is low-mass core-collapse SNe from progenitors of $\sim 8-11 M_{\odot}$ and AIC events. We estimate the Eu yield from an $H$ event by assuming that over the Galactic history of $\sim 10 \mathrm{Gyr}$, the occurrence of $\sim 10^{8}$ such events at a rate of $\sim(100 \mathrm{yr})^{-1}$ enriched $\sim 10^{10} M_{\odot}$ of gas with 
a solar mass fraction $X_{\odot}(\mathrm{Eu})=3.75 \times 10^{-10}$ of Eu (Anders \& Grevesse, 1989). This gives $Y_{H}(\mathrm{Eu}) \sim 3.75 \times 10^{-8} M_{\odot}$. Using $(\mathrm{Sr} / \mathrm{Eu})_{H}=10^{1.41}$ (see Table 3), we obtain

$$
Y_{H}(\mathrm{Sr})=\left(\frac{\mathrm{Sr}}{\mathrm{Eu}}\right)_{H}\left(\frac{A_{\mathrm{Sr}}}{A_{\mathrm{Eu}}}\right) Y_{H}(\mathrm{Eu}) \sim 5.6 \times 10^{-7} M_{\odot}
$$

where $A_{\mathrm{Eu}} \approx 152$ is the mass number of $\mathrm{Eu}$. It can be seen that the yields of CPR nuclei are indeed comparable for an $H$ and $L$ event. This is entirely consistent with these nuclei being produced in the neutrino-driven wind whenever a neutron star is made in any core-collapse SNe. Thus, from the reasonable accord between the predictions of the model and the available data over a wide range of $[\mathrm{Fe} / \mathrm{H}]$ as shown in Figure 17 , it appears that the production of CPR elements per neutron star forming event is rather constant with yields as estimated above.

Using the $H$-yield of Eu estimated above, we can provide some quantitative description of how the extreme $r$-enhancements in HE 2148-1247 discussed in Section 4.5 could have occurred. Taking the radius of this star to be $R$ and the distance from its binary companion to be $d$ when the companion underwent AIC or $\mathrm{O}-\mathrm{Ne}-\mathrm{Mg}$ core collapse to produce the heavy $r$-nuclei, we estimate that a fraction $\sim(R / 2 d)^{2}$ of the $r$-process ejecta would be intercepted by HE 2148-1247. The resulting Eu enrichment would be

$$
\left(\frac{\mathrm{Eu}}{\mathrm{H}}\right) \sim \frac{Y_{H}(\mathrm{Eu}) / A_{\mathrm{Eu}}}{X_{\mathrm{H}} M_{\mathrm{dil}}}\left(\frac{R}{2 d}\right)^{2}
$$

where $X_{\mathrm{H}} \approx 0.76$ is the mass fraction of $\mathrm{H}$ and $M_{\mathrm{dil}}$ is the dilution mass for the intercepted material. Using $Y_{H}(\mathrm{Eu}) \sim 3.75 \times 10^{-8} M_{\odot}, M_{\text {dil }} \sim 0.1 M_{\odot}$, $R \sim R_{\odot}$, and $\log \epsilon(\mathrm{Eu})=0.17$ (Cohen et al., 2003), we obtain $d \sim 23 R_{\odot}$ from the above equation. This appears reasonable and would be consistent with the observational indication that HE 2148-1247 is now in a long-period binary system (Cohen et al., 2003) if its orbit was widened greatly following the AIC or O-Ne-Mg core-collapse event due to, e.g., the kick imparted to the neutron star remnant. In either case, there should be a common-envelope phase during the RGB and AGB evolution of the original primary star.

Finally, we note that the production of CPR nuclei and heavy $r$-nuclei by $H$ events may make important contributions to the inventory of some radioactive nuclei in the ESS. As ${ }^{182} \mathrm{Hf}$ is exclusively made in $H$ events and its inventory in the ESS is measured, we first discuss ${ }^{182} \mathrm{Hf}$. Consider the simple model where uniform production of ${ }^{182} \mathrm{Hf}$ had lasted for a period $T_{\mathrm{UP}}$ and was then followed by an interval $\Delta_{182}$ of no production prior to the formation of the solar system 
(see Section 1). This would result in

$$
\left(\frac{{ }^{182} \mathrm{Hf}}{{ }^{182} \mathrm{~W}_{r}}\right)_{\mathrm{ESS}}=\left(\frac{\bar{\tau}_{182}}{T_{\mathrm{UP}}}\right) \exp \left(-\frac{\Delta_{182}}{\bar{\tau}_{182}}\right)
$$

where ${ }^{182} \mathrm{~W}_{r}$ is the part of ${ }^{182} \mathrm{~W}$ in the solar system contributed by the $r$ process. Using $\left({ }^{182} \mathrm{Hf} /{ }^{180} \mathrm{Hf}\right)_{\mathrm{ESS}}=10^{-4}$ Y Yin et al. (2002); Kleine et al. (2002)], $\left({ }^{180} \mathrm{Hf} /{ }^{182} \mathrm{~W}_{r}\right)_{\odot}=0.0541 / 0.019$ Anders \& Grevesse, 1989; Arlandini et al. $1999)$, and $\tau_{182}=12.8 \mathrm{Myr}$, we obtain $\Delta_{182} \sim 19.2$ Myr for $T_{\mathrm{UP}} \sim 10 \mathrm{Gyr}$.

As mentioned in Section 3 , the neutrino-driven wind makes ${ }^{107} \mathrm{Mo}$, which decays through ${ }^{107} \mathrm{Pd}$ producing ${ }^{107} \mathrm{Ag}$. The model for production of ${ }^{182} \mathrm{Hf}$ by $H$ events discussed above gives

$$
\left(\frac{{ }^{107} \mathrm{Pd}}{{ }^{182} \mathrm{Hf}}\right)_{\mathrm{ESS}, H}=\left(\frac{P_{107}}{P_{182}}\right)_{H}\left(\frac{\bar{\tau}_{107}}{\bar{\tau}_{182}}\right) \exp \left(-\frac{\Delta_{182}}{\bar{\tau}_{107}}+\frac{\Delta_{182}}{\bar{\tau}_{182}}\right),
$$

where $\left(P_{107} / P_{182}\right)_{H}$ is the (number) production ratio of ${ }^{107} \mathrm{Pd}$ to ${ }^{182} \mathrm{Hf}$ for $H$ events and can be estimated as $\sim(1 / 2)(\mathrm{Ag} / \mathrm{Eu})_{H}\left(\mathrm{Eu} /{ }^{182} \mathrm{~W}\right)_{\odot, r}$ (the factor of $1 / 2$ is used as $\mathrm{Ag}$ has an additional isotope $\left.{ }^{109} \mathrm{Ag}\right)$. Taking $(\mathrm{Ag} / \mathrm{Eu})_{H}=$ $10^{0.07}$ (see Table 3), $\left(\mathrm{Eu} /{ }^{182} \mathrm{~W}\right)_{\odot, r}=0.0917 / 0.019$ (Arlandini et al., 1999$)$, and $\bar{\tau}_{107}=9.4 \mathrm{Myr}$, we obtain $\left({ }^{107} \mathrm{Pd} /{ }^{182} \mathrm{Hf}\right)_{\mathrm{ESS}, H} \sim 1.21$. This gives

$$
\left(\frac{{ }^{107} \mathrm{Pd}}{{ }^{108} \mathrm{Pd}}\right)_{\mathrm{ESS}, H}=\left(\frac{{ }^{107} \mathrm{Pd}}{{ }^{182} \mathrm{Hf}}\right)_{\mathrm{ESS}, H}\left(\frac{{ }^{182} \mathrm{Hf}}{{ }^{180} \mathrm{Hf}}\right)_{\mathrm{ESS}}\left(\frac{{ }^{180} \mathrm{Hf}}{{ }^{108} \mathrm{Pd}}\right)_{\odot} \sim 1.78 \times 10^{-5},
$$

where $\left({ }^{180} \mathrm{Hf} /{ }^{108} \mathrm{Pd}\right)_{\odot}=0.0541 / 0.368$ Anders \& Grevesse, 1989) is used. The above result is close to the measured value of $\left({ }^{107} \mathrm{Pd} /{ }^{108} \mathrm{Pd}\right)_{\mathrm{ESS}}=2 \times 10^{-5}$ Kelley \& Wasserburg (1978), see also the recent review by Wasserburg et al. (2006)]. Note that although $L$ events also produce ${ }^{107} \mathrm{Pd}$, their contributions to the ${ }^{107} \mathrm{Pd}$ in the ESS are negligible as the interval between the last $L$ contributions and the formation of the solar system is $\sim 72 \mathrm{Myr}$ based on the ${ }^{129} \mathrm{I}$ data (see Section 1). Therefore, it appears that the $H$ events were responsible for both ${ }^{107} \mathrm{Pd}$ and ${ }^{182} \mathrm{Hf}$ in the ESS. This eliminates the conflict with the data on ${ }^{129} \mathrm{I}$, which is not produced by the $H$ events. However, the broader problems and conflicts regarding ${ }^{53} \mathrm{Mn},{ }^{60} \mathrm{Fe}$, and ${ }^{247} \mathrm{Cm}$ would not be resolved [see the recent review by Wasserburg et al. (2006)].

\subsection{Other models of Galactic chemical evolution}

In the LEGO-block model presented here we have restricted our analysis to a regime where neither low-mass AGB stars nor SNe Ia had contributed sig- 
nificant material to the ISM. The model claims to predict the abundances of all the other elements for any star (without experiencing $s$-process contamination) in this regime given the $\mathrm{Eu}$ and $\mathrm{Fe}$ abundances of the star. It does not seek to provide a detailed or global study of GCE. Models of GCE require assumptions on the rates of formation for a wide range of stellar sources, their yields, and the amount of dilution by mixing with the ISM over time. Many of these models successfully demonstrate general trends over most of Galactic history [e.g., Mathews et al. (1992); Timmes et al. (1995); Travaglio et al. (1999)]. Some models describe the stochastic process of enrichments and can provide a statistical description of abundances in stars [e.g., Ishimaru \& Wanajo (1999); Raiteri et al. (1999); Argast et al. (2004)]. To our knowledge, no GCE models are capable of predicting the abundances of all the other elements in an individual star from its Eu and Fe abundances. We are still open to wagers on the predictions of our LEGO-block model.

\section{Acknowledgments}

The authors thank Hans for many stimulating and illuminating conversations and for being supportive of a strange new approach to the science at hand. We thank Alex Heger and Ken Nomoto for educating us on presupernova evolution and for providing us with Figures 9 and 10, respectively, John Lattanzio for pointing us to literature on AGB stars, and Judy Cohen and Wako Aoki for permitting us to use Figures 12 and 13 , respectively, from their published works. We also thank Marc Kamionkowski for his patience with our unsuccessful efforts in writing a more extensive article with cosmic views on the subject and ask for his forgiveness. Hopefully, this contribution covers much of what we were to write. This work was supported in part by the US Department of Energy under grants DE-FG02-87ER40328 (Y.-Z.Q.) and DE-FG03-88ER13851 (G.J.W.), Caltech Division Contribution 9176(1122).

\section{References}

Aguirre, A., Schaye, J., Kim, T.-S., Theuns, T., Rauch, M., Sargent, W.L.W., 2004. Astrophys. J. 602, 38.

Anders, E., Grevesse, N., 1989. Geochim. Cosmochim. Acta 53, 197.

Aoki, W., Bisterzo, S., Gallino, R., Beers, T.C., Norris, J.E., Ryan, S.G., Tsangarides, S., 2006. Astrophys. J. Lett., in press (astro-ph/0609138).

Aoki, W., Honda, S., Beers, T.C., Kajino, T., Ando, H., Norris, J.E., Ryan, S.G., Izumiura, H., Sadakane, K., Takada-Hidai, M., 2005. Astrophys. J. 632,611 . 
Argast, D., Samland, M., Thielemann, F.-K., Qian, Y.-Z., 2004. Astron. Astrophys. 416, 997.

Arlandini, C., Käppeler, F., Wisshak, K., Gallino, R., Lugaro, M., Busso, M., Straniero, O., 1999. Astrophys. J. 525, 886.

Arnett, W.D., 1996. Supernovae and Nucleosynthesis. Princeton University Press, Princeton.

Barklem, P.S., Christlieb, N., Beers, T.C., Hill, V., Bessell, M.S., Holmberg, J., Marsteller, B., Rossi, S., Zickgraf, F.-J., Reimers, D., 2005. Astron. Astrophys. 439, 129.

Beers, T.C., Preston, G.W., Schectman, S.A., 1992. Astron. J. 103, 1987.

Bethe, H.A., Wilson, J.R., 1985. Astrophys. J. 295, 14.

Burbidge, E.M., Burbidge, G.R., Fowler, W.A., Hoyle, F., 1957. Rev. Mod. Phys. 29, 547.

Busso, M., Gallino, R., Wasserburg, G.J., 1999. Annu. Rev. Astron. Astrophys. 37, 239.

Cameron, A.G.W., 1957. Chalk River Report (see also Pub. Astron. Soc. Pacific 69,201$)$.

Cayrel, R., et al., 2001. Nature 409, 691.

Christlieb, N., Bessell, M.S., Beers, T.C., Gustafsson, B., Korn, A., Barklem, P.S., Karlsson, T., Mizuno-Wiedner, M., Rossi, S., 2003. Nature 419, 904.

Christlieb, N., et al., 2004. Astron. Astrophys. 428, 1027.

Cohen, J.G., Christlieb, N., Qian, Y.-Z., Wasserburg, G.J., 2003. Astrophys. J. 588, 1082.

Cowan, J.J., Sneden, C., Burles, S., Ivans, I.I., Beers, T.C., Truran, J.W., Lawler, J.E., Primas, F., Fuller, G.M., Pfeiffer, B., Kratz, K.-L., 2002. Astrophys. J. 572, 861.

Crawford, J.L., Sneden, C., King, J.R., Boesgaard, A.M., Deliyannis, C.P., 1998. Astron. J. 116, 2489.

Frebel, A., et al., 2005. Nature 434, 871.

Freiburghaus, C., Rembges, J.-F., Rauscher, T., Kolbe, E., Thielemann, F.-K., Kratz, K.-L., Pfeiffer, B., Cowan, J.J., 1999. Astrophys. J. 516, 381.

Freiburghaus, C., Rosswog, S., Thielemann, F.-K., 1999. Astrophys. J. Lett. $525,121$.

Fulbright, J.P., Rich, R.M., Castro, S., 2004. Astrophys. J. 612, 447.

Gallino, R., Arlandini, C., Busso, M., Lugaro, M., Travaglio, C., Straniero, O., Chieffi, A., Limongi, M., 1998. Astrophys. J. 497, 388.

Gallino, R., Busso, M., Picchio, G., Raiteri, C.M., Renzini, A., 1988. Astrophys. J. Lett. 334, 45.

García-Berro, E., Iben, I., Jr., 1994. Astrophys. J. 434, 306.

García-Berro, E., Ritossa, C., Iben, I., Jr., 1997. Astrophys. J. 485, 765.

Harper, C.L., Jr., Jacobsen, S.B. Geochim. Cosmochim. Acta 60, 1131.

Herwig, F., 2005. Annu. Rev. Astron. Astrophys. 43, 435.

Hill, V., et al., 2002. Astron. Astrophys. 387, 560.

Hoffman, R.D., Woosley, S.E., Qian, Y.-Z., 1997. Astrophys. J. 482, 951.

Honda, S., Aoki, W., Ishimaru, Y., Wanajo, S., Ryan, S.G., 2006. Astrophys. 
J. 643, 1180.

Howard, W.M., Goriely, S., Rayet, M., Arnould, M., 1993. Astrophys. J. 417, 713.

Iben, I., Jr., Ritossa, C., García-Berro, E., 1997. Astrophys. J. 489, 772.

Ishimaru, Y., Wanajo, S., 1999. Astrophys. J. Lett. 511, 33.

Ivans, I.I., Simmerer, J., Sneden, C., Lawler, J.E., Cowan, J.J., Gallino, R., Bisterzo, S., 2006. Astrophys. J. 645, 613.

Ivans, I.I., Sneden, C., Gallino, R., Cowan, J.J., Preston, G.W., 2005. Astrophys. J. Lett. 627, 145.

Iwamoto, N., Umeda, H., Tominaga, N., Nomoto, K., Maeda, K., 2005. Science $309,451$.

Johnson, J.A., Bolte, M., 2002. Astrophys. J. 579, 616.

Johnson, J.A., Bolte, M., 2004. Astrophys. J. 605, 462.

Jonsell, K., Barklem, P.S., Gustafsson, B., Christlieb, N., Hill, V., Beers, T.C., Holmberg, J., 2006. Astron. Astrophys. 451, 651.

Käppeler, F., Gallino, R., Busso, M., Picchio, G., Raiteri, C.M., 1990. Astrophys. J. 354, 630.

Karakas, A.I., Lattanzio, J.C., 2004. In: McWilliam, A., Rauch, M. (Eds.), Origin and Evolution of the Elements. Carnegie Observatories, Pasadena, http://www.ociw.edu/ociw/symposia/series/symposium4/proceedings.html.

Kelley, W.R., Wasserburg, G.J., 1978. Geophys. Res. Lett. 5, 1079.

Kleine, T., Münker, C., Mezger, K., Palme, H., 2002. Nature 418, 952.

Kratz, K.-L., Bitouzet, J.-P., Thielemann, F.-K., Möller, P., Pfeiffer, B., 1993. Astrophys. J. 403, 216.

Lee, D.-C., Halliday, A.N., 1995. Nature 378, 771.

Mathews, G.J., Bazan, G., Cowan, J.J., 1992. Astrophys. J. 391, 719.

McWilliam, A., Preston, G.W., Sneden, C., Searle, L., 1995. Astron. J. 109, 2757.

Meyer, B.S., 1995. Astrophys. J. Lett. 449, 55.

Meyer, B.S., Brown, J.S., 1997. Astrophys. J. Suppl. Ser. 112, 199.

Meyer, B.S., Mathews, G.J., Howard, W.M., Woosley, S.E., Hoffman, R.D., 1992. Astrophys. J. 399, 656.

Nomoto, K., 1984. Astrophys. J. 277, 791.

Nomoto, K., 1987. Astrophys. J. 322, 206.

Nomoto, K., Hashimoto, M., 1988. Phys. Rep. 163, 13.

Nomoto, K., Kondo, Y., 1991. Astrophys. J. Lett. 367, 19.

Otsuki, K., Honda, S., Aoki, W., Kajino, T., Mathews, G.J., 2006. Astrophys. J. Lett. 641, 117.

Poelarends, A.J.T., Herwig, F., Langer, N., Heger, A., 2006. Astrophys. J., to be submitted.

Qian, Y.-Z., 2000. Astrophys. J. Lett. 534, 67.

Qian, Y.-Z., Fuller, G.M., Mathews, G.J., Mayle, R.W., Wilson, J.R., Woosley, S.E., 1993. Phys. Rev. Lett. 71, 1965.

Qian, Y.-Z., Vogel, P., Wasserburg, G.J., 1998. Astrophys. J. 494, 285.

Qian, Y.-Z., Wasserburg, G.J., 2001. Astrophys. J. 559, 925. 
Qian, Y.-Z., Wasserburg, G.J., 2002. Astrophys. J. 567, 515.

Qian, Y.-Z., Wasserburg, G.J., 2003. Astrophys. J. 588, 1099.

Qian, Y.-Z., Wasserburg, G.J., 2005. Astrophys. J. 635, 845.

Qian, Y.-Z., Woosley, S.E., 1996. Astrophys. J. 471, 331.

Raiteri, C.M., Villata, M., Gallino, R., Busso, M., Cravanzola, A., 1999. Astrophys. J. Lett. 518, 91.

Reynolds, J.H., 1960. Phys. Rev. Lett. 4, 8.

Ritossa, C., García-Berro, E., Iben, I., Jr., 1996. Astrophys. J.460, 489.

Ritossa, C., García-Berro, E., Iben, I., Jr., 1999. Astrophys. J. 515, 381.

Schramm, D.N., Wasserburg, G.J., 1970. Astrophys. J. 162, 57.

Seeger, P.A., Fowler, W.A., Clayton, D.D., 1965. Astrophys. J. Suppl. Ser. 11, 121.

Sneden, C., Cowan, J.J., Ivans, I.I., Fuller, G.M., Burles, S., Beers, T.C., Lawler, J.E., 2000. Astrophys. J. Lett. 533, 139.

Sneden, C., McWilliam, A., Preston, G.W., Cowan, J.J., Burris, D.L., Armosky, B.J., 1996. Astrophys. J. 467, 819.

Sneden, C., Preston, G.W., Cowan, J.J., 2003. Astrophys. J. 592, 504.

Sneden, C., Preston, G.W., McWilliam, A., Searle, L., 1994. Astrophys. J. Lett. 431, 27.

Sneden, C., et al., 2003. Astrophys. J. 591, 936.

Takahashi, K., Witti, J., Janka, H.-T., 1994. Astron. Astrophys. 286, 857.

Thompson, T.A., Burrows, A., Meyer, B.S., 2001. Astrophys. J. 562, 887.

Thornton, K., Gaudlitz, M., Janka, H.-T., Steinmetz, M., 1998. Astrophys. J. 500, 95 .

Timmes, F.X., Woosley, S.E., Weaver, T.A., 1995. Astrophys. J. Suppl. Ser. 98, 617 .

Travaglio, C., Galli, D., Gallino, R., Busso, M., Ferrini, F., Straniero, O., 1999. Astrophys. J. 521, 691.

Travaglio, C., Gallino, R., Arnone, E., Cowan, J., Jordan, F., Sneden, C., 2004. Astrophys. J. 601, 864.

Van Eck, S., Goriely, S., Jorissen, A., Plez, B., 2001. Nature 412, 793.

Venn, K.A., Irwin, M., Shetrone, M.D., Tout, C.A., Hill, V., Tolstoy, E., 2004. Astron. J. 128, 1177.

Vockenhuber, C., et al., 2004. Phys. Rev. Lett. 93, 172501.

Wanajo, S., Ishimaru, Y., 2006. Nucl. Phys. A, in press.

Wanajo, S., Nomoto, K., Iwamoto, N., Ishimaru, Y., Beers, T.C., 2006. Astrophys. J. 636, 842.

Wanajo, S., Tamamura, M., Itoh, N., Nomoto, K., Ishimaru, Y., Beers, T.C., Nozawa, S., 2003. Astrophys. J. 593, 968.

Wasserburg, G.J., Busso, M., Gallino, R., 1996. Astrophys. J. Lett. 466, 109.

Wasserburg, G.J., Busso, M., Gallino, R., Nollett, K., 2006. Nucl. Phys. A 777,5 .

Wasserburg, G.J., Busso, M., Gallino, R., Raiteri, C.M., 1994. Astrophys. J. $424,412$.

Wasserburg, G.J., Fowler, W.A., Hoyle, F., 1960. Phys. Rev. Lett. 4, 112. 
Westin, J., Sneden, C., Gustafsson, B., Cowan, J.J., 2000. Astrophys. J. 530, 783.

Woosley, S.E., Baron, E., 1992. Astrophys. J. 391, 228.

Woosley, S.E., Heger, A., Weaver, T.A., 2002. Rev. Mod. Phys. 74, 1015.

Woosley, S.E., Hoffman, R.D., 1992. Astrophys. J. 395, 202.

Woosley, S.E., Weaver, T.A., 1995. Astrophys. J. Suppl. Ser. 101, 181.

Woosley, S.E., Wilson, J.R., Mathews, G.J., Hoffman, R.D., Meyer, B.S., 1994. Astrophys. J. 433, 229.

Yin, Q., Jacobsen, S.B., Yamashita, K., Blichert-Toft, J., Télouk, P., Albarède, F., 2002. Nature 418, 949.

\section{A The parable of the key: a story for Hans}

Y.-Z.Q. was heavily involved with teaching in the fall of 2006. In trying to meet the deadline for this volume, G.J.W. traveled to Minneapolis to work on the manuscript. Bad weather conditions left him in Denver overnight. To get some rest, he went out to a more or less nearby Radisson Hotel and caught a few hours' sleep before returning to the Denver Airport early next morning for the remainder of the trip to Minneapolis. Y.-Z.Q. had arranged for him to stay at the Radisson Hotel near the University of Minnesota and met him at the hotel. Upon checking in and getting a card key, they went up to the seventh floor to find Room 729. They searched the seventh floor intensely but could not find a room with that number. G.J.W. was getting quite exhausted so they asked a chambermaid where the room was. She searched and could not find it, either. She asked another maid, but to no avail. Much exercised, G.J.W. insisted that she call the front desk, but she did not know how to make the call. G.J.W. grabbed the phone, got the front desk, and told the clerk of the difficulty. It turned out that Room 729 was not the assigned room. (In fact, it did not exist!) Instead, G.J.W. should go to Room 762. Both Y.-Z.Q. and G.J.W. read a clearly written "729" on the envelope enclosing the key. Nonetheless, off they went to Room 762. This room actually existed. G.J.W. then took the key from the envelope and promptly opened the door to what in fact was his room.

Upon dropping the luggage in the room, G.J.W. (quite exhausted) and Y.Z.Q. went down to get lunch. G.J.W. was rather ticked off that the clerk had clearly written the wrong room number on the key envelope and reported this to the manager. He apologized in the presence of Ben the clerk and asked to see the envelope. Upon seeing it, he immediately said, "this envelope and this key do not belong to this hotel!" He then destroyed the key and re-issued a new one.

Having got no decent sleep, G.J.W. forgot to return the key for Room 729 
where he stayed at the Radisson Hotel in Denver. Being rather disoriented, he randomly pulled the envelope enclosing this key out of a pocket and used it as a guide in search of his room in Minneapolis! Now the question remains, how did the key for a room at the Radisson Hotel in Denver open another room at the Radisson Hotel in Minneapolis? Did the master key to all Radisson Hotel rooms happen to fall into the personal possession of G.J.W.? If so, why was he so stupid as to let the manager take it away and destroy it? Was this just a random event?

Maybe this is how we do science. We use what keys we have and try to get into different rooms and, maybe, sometimes we get into the correct room by accident. G.J.W. still wishes that he did not give up that old key - however, if the new key is the same as the old one, maybe he still has the UNIVERSAL KEY. What luck!!!

Hans would have enjoyed this story.

P.S. Ben got a six-pack of Budweiser for enduring (unjustly) the critical wrath of the junior author. 